\title{
Bases de Gröbner
}

Peterson Pereira de Oliveira

DISSERTAÇÃO APRESENTADA

$\mathrm{AO}$

INSTITUTO DE MATEMÁTICA E ESTATÍSTICA

DA

UNIVERSIDADE DE SÃO PAULO

PARA

OBTENÇÃO DO TÍTULO DE MESTRE

EM

MATEMÁTICA

Área de Concentração: Álgebra

Orientadora: Profa. Dra. Gladys Chalom de Oliveira

Durante a elaboração deste trabalho, o autor recebeu apoio financeiro do CNPq.

São Paulo

2002 


\section{BASES DE GRÖBNER}

Este exemplar corresponde à redação final da dissertação devidamente corrigida e defendida por Peterson Pereira de Oliveira e aprovada pela comissão julgadora.

São Paulo, 17 de setembro de 2002.

Banca Examinadora:

- Profa. Dra. Gladys Chalom de Oliveira (orientadora) (IME-USP)

- Prof. Dr. Eduardo Nascimento Marcos (IME-USP)

- Prof. Dr. Arnaldo Mandel (IME-USP) 


\section{Resumo}

Neste trabalho, estudamos a Teoria das Bases de Gröbner para álgebras não comutativas. Além disso, vimos que uma $\mathcal{K}$-álgebra com unidade, que possui a teoria das bases de Gröbner, é isomorfa a um quociente de Álgebra de Caminhos. Definimos a álgebra estendida por laços, que é uma generalização para o caso não comutativo do processo de homogeneização. Expomos aqui o resultado principal: Seja $F$ um subconjunto de uma álgebra de caminhos $\Lambda$ e seja $\mathcal{G} \subset \Lambda^{\prime}$ homogêneo, onde $\Lambda^{\prime}$ é a álgebra $\Lambda$ estendida por laços. Se $\mathcal{G}$ é uma base de Gröbner para $\left\langle F^{*}\right\rangle$, então $\mathcal{G}_{*}$ é uma base de Gröbner para $\langle F\rangle$. 


\section{Abstract}

In this work, we study Gröbner Bases Theory to non commutative algebras. Moreover, we see that a $\mathcal{K}$-algebra with unity and with Gröbner bases theory is isomorphic to a quocient of path algebras. We define algebra extended by loops, as a generalization of the homogenization process to non commutative case. Here, we present the main result: Let $F$ be a subset of the path algebra $\Lambda$ and let $\mathcal{G} \subset \Lambda^{\prime}$ be homogeneous, where $\Lambda^{\prime}$ is the algebra $\Lambda$ extended by loops. If $\mathcal{G}$ is a Gröbner basis to $\left\langle F^{*}\right\rangle$, then $\mathcal{G}_{*}$ is a Gröbner basis to $\langle F\rangle$. 
Aos meus queridos pais,

Elifas e Zilma. 


\section{Introdução}

Nesse trabalho apresentamos um pouco sobre a teoria das Bases de Gröbner apresentadas em [7], [9] e [10], tendo feito um estudo detalhado desses artigos. Como estamos interessados em estudar essa teoria no caso não comutativo, surgiu, em discussão (com o autor de [9] e [10]) a idéia de generalizar o processo de homogeneização do caso comutativo (veja [3], cap.10) para o caso não comutativo.

Começamos aqui com a teoria para espaços vetoriais (Capítulo 2) e estendemos esses resultados para $\mathcal{K}$-álgebras, após ter introduzido o conceito de ordem admissível. Nos procedimentos Algoritmo da Divisão e Construção de Bases de Gröbner, ambos para o caso não comutativo, descritos em [9], houve a necessidade de se fazer algumas correções para que fornecessem o resultado esperado.

Ainda no Capítulo 2 (seção 5), temos alguns resultados que mostram em que situações podemos garantir a existência de uma base de Gröbner finita.

Na seção Por que Álgebras de Caminhos? (seção 3.1), podemos ver a importância dessas álgebras no estudo das bases de Gröbner (veja [7] e [9]). Nesta seção, temos um dos principais resultados do trabalho: uma $\mathcal{K}$-álgebra com unidade que possui a teoria de base de Gröbner, ou seja, possui uma $\mathcal{K}$-base multiplicativa com uma ordem admissível nessa base, é isomorfa ao quociente de uma álgebra de caminhos.

Uma questão que ainda está aberta é: quais são as condições necessárias 
e suficientes sobre um ideal I, para que o quociente $\mathcal{K} \mathcal{Q} / I$ tenha a teoria de bases de Gröbner?, sabemos apenas que se o ideal for 2-nomial, isto é, se puder ser gerado por elementos da forma $p, q-r$, com $p, q, r$ pertencentes a. $\mathcal{K}$-base de $\mathcal{K} \mathcal{Q}$, então o quociente $\mathcal{K} \mathcal{Q} / I$ possui uma base multiplicativa, o que não implica em ter uma ordem admissível nessa base. Os resultados apresentados até este momento podem ser encontrados em [7], [9] e [10].

Na seção 3.2, temos a generalização dos resultados encontrados em [3] (cap.10) para álgebras de caminhos, $\mathcal{K} \mathcal{Q} / I$. 


\section{Sumário}

1 Preliminares 4

1.1 Categorias e Funtores . . . . . . . . . . . . . . . 4

1.2 Anéis e Módulos . . . . . . . . . . . . . . . . 5 5

1.2 .1 Anéis . . . . . . . . . . . . . . . 5

1.2 .2 Módulos . . . . . . . . . . . . . . . . . 7

1.3 Álgebras . . . . . . . . . . . . . . . . . . . . 8

1.3.1 Álgebras de Caminhos ... . . . . . . . . . 10

1.4 Ordem . . . . . . . . . . . . . . . . . . . 13

2 Bases de Gröbner $\quad 15$

2.1 Bases de Gröbner Lineares . . . . . . . . . . . . . . . 15

2.2 Bases de Gröbner para K-Álgebras . . . . . . . . . . . . . 19

2.3 Algoritmo da Divisão . . . . . . . . . . . . . . . . . . . 22

2.4 Relações de Sobreposição . . . . . . . . . . . . . . . . 26

$2.5 \mathcal{K}$-álgebras de Dimensão Finita . . . . . . . . . . . . . 32

3 Bases de Gröbner e Álgebra de Caminhos 35

3.1 Por que Álgebras de Caminhos? . . . . . . . . . . . . . . . . . 35

3.2 Homogeneização . . . . . . . . . . . . . . . . 43 


\section{Capítulo 1}

\section{Preliminares}

Antes de iniciarmos as definições e resultados sobre a Teoria de Bases de Gröbner, veremos alguns conceitos básicos para um entendimento melhor dessa teoria (tais conceitos podem ser encontrados com mais detalhes em [1] e [2]).

\subsection{Categorias e Funtores}

Uma categoria $\mathcal{C}$ consiste de uma classe de objetos, Obj $\mathcal{C}$, e para cada par ordenado de objetos, $(A, B)$, tem-se o conjunto de morfismos, $\mathcal{C}(A, B)$ (eventualmente vazio), tais que, se $(A, B) \neq(C, D)$, então $\mathcal{C}(A, B) \cap \mathcal{C}(C, D)=\varnothing$ e uma aplicação $\mathcal{C}(A, B) \times \mathcal{C}(B, C) \rightarrow \mathcal{C}(A, C)$, denotada por $(f, g) \mapsto g f$, satisfazendo os seguintes axiomas:

(1) Para cada objeto $A$, existe um morfismo identidade $1_{A} \in \mathcal{C}(A, A)$ tal que $f 1_{A}=f$ para todo $f \in \mathcal{C}(A, B)$ e $1_{A} g=g$ para todo $g \in \mathcal{C}(C, A)$;

(2) Se $A, B, C, D$ são objetos da categoria $\mathcal{C} \operatorname{com} \alpha \in \mathcal{C}(A, B), \beta \in \mathcal{C}(B, C)$, $\gamma \in \mathcal{C}(C, D)$, então $\gamma(\beta \alpha)=(\gamma \beta) \alpha$ (propriedade associativa) 
Em uma categoria $\mathcal{C}$, um morfismo $f \in \mathcal{C}(A, B)$ é chamado de isomorfismo se existe $g \in \mathcal{C}(B, A)$ tal que $f g=1_{B}$ e $g f=1_{A}$. Quando existe um isomorfismo $f \in \mathcal{C}(A, B)$ diz-se que $A$ é isomorfo a $B$ e denota-se $A \cong B$.

Um morfismo $f \in \mathcal{C}(A, B)$ é chamado monomorfismo em $\mathcal{C}$ (respectivamente epimorfismo) se quaisquer que sejam $g_{1}, g_{2} \in \mathcal{C}(C, A)$ (respectivamente $\in \mathcal{C}(A, C)$ ), sempre que $f g_{1}=f g_{2}$ (respectivamente $g_{1} f=g_{2} f$ ) implicar $g_{1}=g_{2}$.

Dizemos que $X$ é um objeto zero de uma categoria $\mathcal{C}$ se para qualquer objeto $Y \in \mathrm{Obj} \mathcal{C}$, não vazio, tem-se: $\mathcal{C}(X, Y)$ e $\mathcal{C}(Y, X)$ são conjuntos unitários, este objeto é único a menos de isomorfismo e vamos denotá-lo por 0 .

Seja $\mathcal{C}$ uma categoria e $A, B$ objetos em $\mathcal{C}$. Um objeto $P$ na categoria $\mathcal{C}$ é dito projetivo se para qualquer epimorfismo $g: A \rightarrow B$ e morfismo $h: P \rightarrow B$, existe um morfismo $s: P \rightarrow A$ tal que $g s=h$.

\subsection{Anéis e Módulos}

\subsubsection{Anéis}

Um anel $R$ é um conjunto não vazio munido de duas operações binárias, chamadas adição $(+)$ e multiplicação (.), tais que:

(1) $R$ é um grupo abeliano com relação à adição, isto é, $(R,+)$ é um grupo abeliano.

(2) $R$ é um semigrupo com relação à multiplicação, isto é, $(R$, .) é um semigrupo.

(3) A operação de multiplicação é distributiva sobre a adição, ou seja, para todo $z, x, y \in R$, temos $z(x+y)=z x+z y$ e $(z+x) y=z y+x y$.

(4) $R$ contém unidade, isto é, existe $1 \in R$ tal que $1 . x=x .1=x$ para todo $x \in R$. 
Seja $S$ um subconjunto de um anel $R$. Dizemos que $S$ é um subanel se $S$ é fechado com relação à adição e à multiplicação e se contém a unidade de $R$. Um ideal à esquerda (respectivamente à direita) é um subconjunto $I$ de $R$ com as seguintes propriedades:

$\forall i, j \in I$ tem-se $i+j \in I$;

$\forall r \in R$ e $\forall i \in I$ tem-se $r i \in I$ (respectivamente $i r \in I$ ).

Chamamos de ideal bilateral de $R$ ao ideal que é simultaneamente ideal à esquerda e à direita.

Sejam $R$ e $S$ anéis. Uma transformação $f: R \rightarrow S$ é um homomorfismo de anéis com unidade quando $f$ preserva adição, multiplicação e unidade. Em outras palavras, $f$ satisfaz as condições:

- $f(x+y)=f(x)+f(y)$

- $f(x \cdot y)=f(x) \cdot f(y)$

- $f\left(1_{R}\right)=1_{S}$

A composição de homomorfismos de anéis é também um homomorfismo de anel. Chamamos de endomorfismos aos homomorfismos que vão de um anel em si mesmo. Um isomorfismo entre os anéis $R$ e $S$, de acordo com a definição dada na página 5 , é um homorfismo que é bijetivo. Neste caso, costumamos dizer que $R$ e $S$ são isomorfos como anéis.

Um divisor de zero à esquerda (respectivamente à direita) é um elemento $r \in R$ para. o qual existe $s \in R \backslash\{0\}$ tal que $r . s=0$ (respectivamente $s . r=0$ ). E um elemento invertível à esquerda (respectivamente à direita) em $R$ é um elemento $r \in R$ para o qual existe $s \in R$ tal que r.s $=1$ (respectivamente $s . r=1)$. Um anel em que todos os elementos não nulos são invertíveis à esquerda e à direita é chamado de anel com divisão. 


\subsubsection{Módulos}

Seja $R$ um anel. Um $R$-módulo à esquerda é um grupo abeliano $M$, que denotaremos aditivamente, sobre o qual existe uma ação linear de $R$, isto é, existe uma transformação $(-,-): R \times M \rightarrow M$, denotada por $(r, m)=r m$, que satisfaz:

(1) $(r+s) m=r m+s m$

(2) $r(m+n)=r m+r n$

(3) (r.s) $m=r(s m)$

(4) $1 . m=m$

para todos $r, s \in R$ e $m, n \in M$.

Seja $R$ um anel. Considere as aplicações $\lambda, \rho: R \times R \rightarrow R$ definidas por: $\lambda(a, x)=a x$ e $\rho(a, x)=x a . \quad R$ munido de sua operação aditiva e a multiplicação determinada por $\lambda$ (respectivamente $\rho$ ) é um $R$-módulo à esquerda (respectivamente à direita) o qual denotaremos por ${ }_{R} R$ (respectivamente $R_{R}$ ).

Sejam $M$ e $N R$-módulos. Uma aplicação $f: M \rightarrow N$ é um homomorfismo de $R$-módulos se preserva a operação de soma e a ação de $R$, ou seja, se satisfaz as condições:

(1) $f(m+n)=f(m)+f(n) \forall m, n \in M$

(2) $f(r m)=r f(m) \forall m \in M$ e $r \in R$

Neste caso também dizemos que $f$ é $R$-linear.

Observe que a composição de dois homomorfismos de $R$-modulos é novamente um homomorfismo de $R$-módulos.

$\mathrm{O}$ anel dos endomorfismos de um módulo $M$ é o anel dos homomorfismos de módulo em si mesmo. 
Um isomorfismo de módulo é um homomorfismo de módulos $f: M \rightarrow N$, que é bijetivo. Neste caso dizemos que $M$ e $N$ são isomorfos.

Um subconjunto $N$ de $M$ é um submódulo de $M$, se $N$ é um subgrupo aditivo de $M$ e $r n \in N$ para todo $r \in R$ e $n \in N$. (Os submódulos de ${ }_{R} R$ são os ideais à esquerda de $R$ )

Seja $f: M \rightarrow N$ um homomorfismo de $R$-módulos. Definem-se o núcleo e a imagem de $f$ da seguinte forma:

- Núcleo de $f:=\operatorname{Ker}(f)=\{m \in M: f(m)=0\}$

- Imagem de $f:=\operatorname{Im}(f)=\{n \in N: f(m)=n$ para algum $m \in M\}$

É de imediata verificação que $\operatorname{Ker}(f)$ e $\operatorname{Im}(f)$ são submódulos de $M$ e $N$, respectivamente.

Na categoria de módulos sobre um anel $R$, a definição dada na seção 1.1 para monomorfismos (respectivamente epimorfismos) pode ser dada da seguinte forma: um morfismo de $R$-módulos $f: M \rightarrow N$ é um monomorfismo (epimorfismo) se $K \operatorname{er}(f)=\{0\}$, ou seja, se for injetor (respectivamente $\operatorname{Im}(f)=N$, ou seja, se for sobrejetor).

Uma sequência de morfismos

$$
\cdots \stackrel{f_{3}}{\longrightarrow} M_{2} \stackrel{f_{2}}{\longrightarrow} M_{1} \stackrel{f_{1}}{\longrightarrow} M_{0} \stackrel{f_{0}}{\longrightarrow} M \longrightarrow 0
$$

é exata quando $\operatorname{Im}\left(f_{i}\right)=\operatorname{Ker}\left(f_{i-1}\right)$, para todo $i>0$ e $f_{0}$ for sobrejetor. Quando os módulos $M_{i}$ são projetivos, dizemos que a sequência exata é uma resolução projetiva de $M$. Note que, nesse caso, $M \cong M_{0} / \operatorname{Im}\left(f_{1}\right)$. Como todo módulo é imagem homomórfica de um módulo livre, a resolução projetiva sempre existe. Para mais detalhes veja [4].

\section{3 Álgebras}

Seja $R$ um anel comutativo. Uma $R$-álgebra $A$ é um $R$-módulo munido de uma operação de multiplicação (.), que satisfaz: 
(1) $(A,$.$) é um semigrupo.$

(2) A operação de multiplicação é distributiva sobre a adição de $A$ vista como $R$-módulo.

Além disso, $R$ age centralmente em $A$, ou seja,

$$
r(a b)=(r a) b=a(r b)
$$

para todo $a, b \in A$ e $r \in R$.

Se a $R$-álgebra $A$ possui unidade, dizemos que a $R$-álgebra $A$ é uma álgebra com unidade e temos o seguinte homomorfismo de anéis:

$$
\begin{aligned}
\varepsilon: R & \longrightarrow A \\
r & \longmapsto \varepsilon(r)=r 1
\end{aligned}
$$

Quando $R$ é um corpo, uma base para a $R$-álgebra $A$, é uma base para $A$ vista como módulo, ou seja, como $R$ espaço vetorial. Se $A$ admite uma $R$-base finita, dizemos que $A$ tem dimensão finita sobre $R$.

Sejam $A$ e $B R$-álgebras. Uma transformação $f: A \rightarrow B$ é um homomorfismo de $R$-álgebras se satisfaz:

(1) $f(a+b)=f(a)+f(b) \forall a, b \in A$

(2) $f(x a)=x f(a) \forall a \in A, \forall x \in R$

(3) $f(a b)=f(a) f(b) \forall a, b \in A$

(4) Se $A$ e $B$ são álgebras com unidade, então $f\left(1_{A}\right)=1_{B}$.

Um homomorfismo de $R$-álgebras que for sobrejetor e injetor é um isomorfismo de $R$-álgebras, neste caso dizemos que $A$ e $B$ são álgebras isomorfas e escreveremos $A \cong B$. Relembramos também que aqui, nesta categoria, os homomorfismos injetores são monomorfismos e os sobrejetores são epimorfismos. 


\subsection{1 Álgebras de Caminhos}

Nos exemplos que serão dados neste trabalho, utilizaremos apenas as álgebras de caminhos. Esse uso não é de forma nenhuma uma coincidência. Mais adiante, veremos que as álgebras de caminhos são inevitáveis no estudo da teoria de bases de Gröbner.

Agora vamos introduzir as noções de quiver e de álgebra de caminhos. Um quiver é um sistema $\mathcal{Q}\left(\mathcal{Q}_{0}, \mathcal{Q}_{1}, o, t\right)$, onde $\mathcal{Q}_{0}$ é um conjunto (cujos elementos são chamados de vértices), $\mathcal{Q}_{1}$ é um conjunto (cujos elementos são chamados de flechas), e cada uma das aplicações $o, t: \mathcal{Q}_{1} \rightarrow \mathcal{Q}_{0}$ associa a cada flecha um vértice. Se $\alpha \in \mathcal{Q}_{1}, o(\alpha)$ é chamado de origem de $\alpha$ e $t(\alpha)$ é chamado de término de $\alpha$. Um quiver é dito finito se ambos os conjuntos $\mathcal{Q}_{0}$ e $\mathcal{Q}_{1}$ são finitos.

Neste trabalho assumiremos que todo quiver $\mathcal{Q}$ é finito.

DeFINIÇĀo. 1.1 Um caminho $\gamma$ de comprimento $n, n>0$, no quiver $\mathcal{Q}$ é uma sequência de flechas $\gamma=\alpha_{1} \ldots \alpha_{n}$ tal que $t\left(\alpha_{i}\right)=o\left(\alpha_{i+1}\right)$ para todo $i=1, \cdots, n-1$ (isto é, $\alpha_{i} \in \mathcal{Q}_{1}$ para todo $i \in\{1, \cdots, n\}$ ). A cada vértice $i \in \mathcal{Q}_{0}$ associamos também um caminho, chamado caminho trivial ou de comprimento nulo, denotado por $e_{i}$.

Definiçāo. 1.2 Dado um caminho não trivial $\gamma=\alpha_{1} \ldots \alpha_{n}$ definimos $o(\gamma)=$ $o\left(\alpha_{1}\right)$ e $t(\gamma)=t\left(\alpha_{n}\right)$. No caso em que o $(\gamma)=t(\gamma)$ dizemos que $\gamma$ é um circuito orientado.

Definiçāo. 1.3 Dados $i, j \in \mathcal{Q}_{0}$ dizemos que $i$ e $j$ estão unidos por aresta se existe $\alpha \in \mathcal{Q}_{1}$ tal que $o(\alpha)=i$ e $t(\alpha)=j$ ou $o(\alpha)=j$ e $t(\alpha)=i$. Um quiver é conexo quando para quaisquer dois vértices $i$ e $j$ em $\mathcal{Q}_{0}$ a seguinte condição é verificada:

- Sempre que $i \neq j$ existe uma sequência de vértices $i=l_{1}, \cdots, l_{n}=j$ tais que $l_{k}$ e $l_{k+1}$ estão unidos por aresta, para todo $k \in\{1, \cdots, n-1\}$. 
Seja $\mathcal{K}$ um corpo. Consideremos $\mathcal{K} \mathcal{Q}$ o $\mathcal{K}$-espaço vetorial tendo como base o conjunto de todos os caminhos de $\mathcal{Q}$. É importante notarmos que $\mathcal{K} \mathcal{Q}$ tem estrutura de $\mathcal{K}$-álgebra, quando definimos a operação de multiplicação (.) de caminhos da seguinte forma:

$$
\alpha \cdot \beta=\left\{\begin{array}{cc}
0 & \text { se } o(\beta) \neq t(\alpha) \\
\alpha \beta & \text { se } o(\beta)=t(\alpha)
\end{array}\right.
$$

Definiçāo. 1.4 Sejam $\mathcal{K}$ um corpo e $\mathcal{Q}$ um quiver. Denotamos por $J o$ ideal de $\mathcal{K} \mathcal{Q}$ gerado por todas as flechas de $\mathcal{Q}_{1}$. Dizemos que um ideal $X$ de $\mathcal{K Q}$ é admissivel quando satisfaz:

- $X \subset J^{2}$

- $J^{n} \subset X$ para algum $n \in \mathbb{N}$

Uma relação em $\mathcal{Q}$ é uma combinação linear de caminhos de comprimento maior ou igual a dois com mesma origem e mesmo término.

Um importante resultado com relação a estes conceitos é a proposição abaixo cuja demonstração pode ser encontrada em [1].

Proposiçāo 1.5 Seja I um ideal admissível da $\mathcal{K}$-álgebra $\mathcal{K} \mathcal{Q}$. Então existe um conjunto finito de relações $\rho_{1}, \cdots, \rho_{t}, t \in \mathbb{N}$ tal que $I=\left\langle\rho_{1}, \cdots, \rho_{t}\right\rangle$.

Definiçāo. 1.6 Seja $\rho=\left\{\rho_{1}, \cdots, \rho_{t}\right\}$ um conjunto de relações em $\mathcal{K} \mathcal{Q}$. Dizemos que $(\mathcal{Q}, \rho)$ é um quiver com relações e a $\mathcal{K}$-álgebra quociente de $\mathcal{K} \mathcal{Q}$ pelo ideal $\langle\rho\rangle$ é chamada algebra de caminhos de $(\mathcal{Q}, \rho)$ sobre o corpo $\mathcal{K}$, que denotaremos simplesmente por $\mathcal{K} \mathcal{Q} /\langle\rho\rangle$.

Lembramos que uma álgebra $A$ é básica quando ${ }_{A} A=P_{1} \coprod \cdots \coprod P_{s}$, onde $P_{i}$ é $A$-módulo projetivo indecomponível e $P_{i} \not P_{j}$ sempre que $i \neq j$ para todo $i, j \in\{1, \cdots, s\}$. O próximo resultado nos mostra como as álgebras de caminhos são importantes, e podemos encontrar sua demonstração em [1]. 
$\mathrm{P}_{\text {roposiçāo }} 1.7$ (Teorema de Gabriel) Seja $\mathcal{K}$ um corpo algebricamente fechado, $\in \Lambda$ uma $\mathcal{K}$-álgebra conexa, básica e de dimensão finita. Então existe um quiver $\mathcal{Q}$ e um conjunto de relaçôes $\rho$, tal que $\langle\rho\rangle$ seja um ideal admissivel de $\mathcal{K} \mathcal{Q}$, de modo que $\Lambda$ é isomorfo à álgebra de caminhos $\mathcal{K} \mathcal{Q} /\langle\rho\rangle$.

O quiver $\mathcal{Q}$ associado à $\Lambda$ recebe o nome de quiver ordinário de $\Lambda$.

Seja $\Lambda$ uma $\mathcal{K}$-álgebra de dimensão finita, indecomponível e básica, e seja. $E=\left\{e_{1}, \cdots, e_{n}\right\}$ um sistema completo de idempotentes ortogonais e primitivos de $\Lambda$. A determinação do quiver ordinário de $\Lambda$ é realizada tomando um conjunto (de vértices) em bijeção com $E$, e estipulando que entre os vértices $i$ e $j$ existem exatamente $\operatorname{dim}_{\mathcal{K}}\left[e_{j}\left(\operatorname{rad} \Lambda / \operatorname{rad}^{2} \Lambda\right) e_{i}\right]$ flechas.

Seja $\mathcal{Q}$ um quiver. Uma representação de $\mathcal{Q}$ é dado pelo sistema

$$
V=\left(\left(V_{i}\right)_{i \in \mathcal{Q}_{0}},\left(f_{\alpha}\right)_{\alpha \in \mathcal{Q}_{1}}\right),
$$

onde para cada $i \in \mathcal{Q}_{0}, V_{i}$ é um $\mathcal{K}$-espaço vetorial (não necessariamente de dimensão finita) e $f_{\alpha}$ é uma transformação $\mathcal{K}$-linear de $V_{o(\alpha)}$ para $V_{t(\alpha)}$. Dado um caminho não trivial $\gamma=\alpha_{1} \ldots \alpha_{s}, s \in \mathbb{N}$, de $i$ a $j$ em $\mathcal{Q}$, podemos definir $V(\gamma)$ como a transformação linear de $V_{i}$ para $V_{j}$ dada pela composta $f_{\alpha_{s}} \ldots f_{\alpha_{1}}$ e estendendo esta definição para uma combinação linear de caminhos.

Dizemos que uma representação $V$ satisfaz uma relação $r$ se $V(r)=0$, e dizemos que $V$ satisfaz um sistema de relações $\rho$ se $V$ satisfizer cada relação $r \in \rho$. Uma representação de um quiver com relações $(\mathcal{Q}, \rho)$ é uma representação de $\mathcal{Q}$ que satisfaz as relações de $\rho$.

Definiçā̃o. 1.8 Sejam $V=\left(\left(V_{i}\right)_{i \in \mathcal{Q}_{0}},\left(f_{\alpha}\right)_{\alpha \in \mathcal{Q}_{1}}\right)$ e $W=\left(\left(W_{i}\right)_{i \in \mathcal{Q}_{0}},\left(g_{\alpha}\right)_{\alpha \in \mathcal{Q}_{1}}\right)$ duas representações de $\mathcal{Q}$, um morfismo $\phi: V \rightarrow W$ é uma família de transformações $\mathcal{K}$-lineares $\phi=\left(\phi_{i}\right)_{i \in \mathcal{Q}_{0}}$ tal que, para cada $\alpha \in \mathcal{Q}_{1}$, o seguinte 
diagrama comuta:

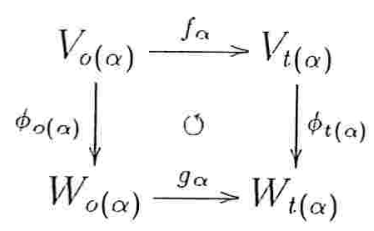

A composição de morfismos é definida coordenada a coordenada. Desta forma, fica definida a categoria das representaçôes de $(\mathcal{Q}, \rho)$.

Dada uma $\mathcal{K}$-álgebra $\Lambda$ de dimensão finita, indecomponível, básica, e sendo $\mathcal{K}$ algebricamente fechado, pelo teorema de Gabriel $\Lambda$, é isomorfa a uma álgebra de caminhos da forma $\mathcal{K} \mathcal{Q} /\langle\rho\rangle$, nestas condições, a categoria construida da forma acima e a categoria dos módulos à direita, que são finitamente gerados sobre a álgebra $\Lambda$, são equivalentes.

\subsection{Ordem}

A escolha de uma boa ordem é fundamental para o estudo da teoria das bases de Gröbner. Mas antes, precisamos de alguns conceitos básicos a respeito de ordem.

DefiniçĀo. 1.9 Uma ordem parcial em um conjunto não vazio $X$ é uma relação transitiva, antissimétrica e reflexiva em $X$, que denotaremos por $\leq$ e escreveremos $x \leq y$, com $x, y \in X$ para indicar que o par ordenado $(x, y)$ pertence a ordem.

Diremos ainda que um conjunto $X$ é parcialmente ordenado se existe uma. ordem parcial em $X$.

Dado um conjunto $X$, parcialmente ordenado por $\leq$, pode ocorrer que para todo $x, y \in X, x \leq y$ ou $y \leq x$. Neste caso, dizemos que $\leq$ é uma ordem total em $X$ e que $X$ é totalmente ordenado. Chamaremos aqui um conjunto totalmente ordenado de cadeia.

Utilizaremos ainda a seguinte notação: 
i) $x \geq y$ se $y \leq x$

ii) $x<y$ se $x \leq y$ e $x \neq y$;

iii) $x>y$ se $y \leq x$ e $x \neq y$.

Definiçāo. 1.10 Seja $X$ um conjunto parcialmente ordenado. Um elemento $a \in X$ é dito mínimo se $a \leq x$ para todo $x \in X$.

Temos ainda que $a \in X$ é dito minimal se $x \leq$ a implica $x=$ a para todo $x \in X$.

Por fim, diremos que $a \in X$ é limitante inferior de um subconjunto $E$ de $X$ se $a \leq x$ para qualquer $x \in E$.

Definição. 1.11 Dizemos que $\leq$ é uma boa ordem em $X$, se é uma ordem total em $X$ e todo subconjunto não vazio possui um elemento minimal.

O próximo resultado é fundamental para o restante do trabalho.

Teorema 1.12 Seja $X$ um conjunto. Então $\leq e ́$ uma boa ordem em $X$ se e somente se $\leq$ é uma ordem total e para cada cadeia descendente de elementos de $X, b_{1} \geq b_{2} \geq \cdots$ existe algum $N \in \mathbb{N}^{*}$ tal que $b_{N}=b_{N+1}=\cdots$. 


\section{Capítulo 2}

\section{Bases de Gröbner}

As bases de Gröbner, que aparecem inicialmente no trabalho de Buchberger, em 1965, tem como idéia principal o de providenciar um método sistemático de construir um conjunto de geradores para ideais de um anel (de polinômios) e decidir se determinado polinômio pertence ou não a esse ideal. Esse método é particularmente interessante do ponto de vista computacional. As definições e resultados dadas nesse capítulo podem ser encontrados em [9] e [10].

\subsection{Bases de Gröbner Lineares}

Nesta seção vamos considerar somente espaços vetoriais. As idéias introduzidas aqui servirão de base para o restante do trabalho. E trataremos de a.lgumas propriedades das bases de Gröbner nesse caso.

Seja $\mathcal{K}$ um corpo e $V$ um $\mathcal{K}$-espaço vetorial. Fixemos, para $V$, uma base $\mathcal{B}=\left\{b_{i}\right\}_{i \in \mathcal{I}}$, onde $\mathcal{I}$ é um conjunto de índices.

Como $\mathcal{B}$ é uma base de $V$, então para cada $v \in V$, existe uma família $\left(\lambda_{i}\right)_{i \in \mathcal{I}}$ tal que $v=\sum_{i \in \mathcal{I}} \lambda_{i} b_{i}$, onde $\lambda_{i}=0$, exceto para um número finito de indices. 
$\operatorname{Se} v=\sum_{i \in \mathcal{I}} \lambda_{i} b_{i}$, dizemos que $b_{i}$ ocorre em $v$ se $\lambda_{i} \neq 0$.

Definiçāo. 2.1 Se $\mathcal{B}=\left\{b_{i}\right\}_{i \in \mathcal{I}}$ é uma base do espaço vetorial $V$, com uma boa ordem $>$ em $\mathcal{B}$, e se $v=\sum_{i \in \mathcal{I}} \lambda_{i} b_{i}$ é não nulo, dizemos que $b_{i}$ é o maior elemento de $v$ se $b_{i}$ ocorre em $v e b_{i} \geq b_{j}$ para todo $b_{j}$ ocorrendo em $v$. Denotaremos o maior elemento $b_{i}$ de $v$ por Tip $(v)$ e o seu coeficiente $\lambda_{i}$ por $\operatorname{CTip}(v)$.

$O$ conjunto dos elementos $b_{i} \in \mathcal{B}$ tais que $b_{i}$ ocorre em $v$ é dito suporte de $v$, denotado por $\operatorname{supp}(v)$.

Se $X$ é um subconjunto de $V$, definimos por

(i) $\operatorname{Tip}(X)=\{b \in \mathcal{B}: b=\operatorname{Tip}(x)$ para algum $0 \neq x \in X\}$

(ii) $\operatorname{NonTip}(X)=\mathcal{B} \backslash \operatorname{Tip}(X)$

Assim, ambos, Tip $(X)$ e $\operatorname{NonTip}(X)$ são subconjuntos de $\mathcal{B}$ e dependem da escolha da boa ordem de $\mathcal{B}$.

Denotamos por $\operatorname{Span}(X)$ o espaço vetorial gerado pelo conjunto $X$.

O próximo resultado será bastante usado ao longo do trabalho.

Teorema 2.2 Seja V um espaço vetorial sobre o corpo $\mathcal{K}$, com base $\mathcal{B}$. Seja $>$ uma boa ordem em $\mathcal{B}$. Suponha que $W$ é subespaço de $V$, então

$$
V=W \oplus \operatorname{Span}(\operatorname{NonTip}(W))
$$

Prova. Seja $W^{\prime}=\operatorname{Span}(\operatorname{NonTip}(W))$. Primeiramente vamos mostrar que $W \cap W^{\prime}=\{0\}$.

Seja $x \in W^{\prime} \backslash\{0\}$. Se $x \in W$, então $\operatorname{Tip}(x) \in \operatorname{Tip}(W)$. No entanto, $\operatorname{Tip}(x) \in \operatorname{NonTip}(W)$ pois $x \in W^{\prime}$, e assim obteríamos uma contradição.

Agora, mostraremos que $V=W+W^{\prime}$. Aqui usaremos o fato da boa ordenação em $\mathcal{B}$. 
Seja $v \in V$ tal que Tip $(v)$ é minimal com respeito a propriedade de $v \notin W+W^{\prime}$, mostraremos que isso nos levará a uma contradição.

Considere $\operatorname{Tip}(v)=b$ e $\alpha=\operatorname{CTip}(v)$. Note que Tip $(v-\alpha b)<\operatorname{tip}(v)$. Assim $w+w^{\prime}=v-\alpha b \in W+W^{\prime}$, pela condição de minimalidade, com $w \in W$ e $w^{\prime} \in W^{\prime}$.

Se $b \in \operatorname{NonTip}(W)$, teremos $w^{\prime}+\alpha b \in W^{\prime}$ e assim,

$$
v=w+\left(w^{\prime}+\alpha b\right)
$$

o que não pode acontecer.

Por outro lado, se $b \in \operatorname{Tip}(W)$, então existe $z \in W$ tal que Tip $(z)=b$, seja $\beta=\operatorname{CTip}(z)$. Então Tip $(v-(\alpha / \beta) z)<\operatorname{Tip}(v)$. Novamente, pela condição de minimalidade, temos que $v-(\alpha / \beta) z=w+w^{\prime} \operatorname{com} w \in W \mathrm{e}$ $w^{\prime} \in W^{\prime}$.

Logo, temos uma contradição, pois

$$
v=(w+(\alpha / \beta) z)+w^{\prime} \in W+W^{\prime}
$$

Do teorema 2.2 podemos ver que todo vetor não nulo $v \in V$ pode ser escrito de forma única como $w_{v}+N(v)$, onde $N(v) \in \operatorname{Span}(N o n T i p(W))$ e $w_{v} \in W$.

Definiçāo. 2.3 Chamamos $N(v)$ a forma normal de $v$.

Seja $W$ um subespaço de $V$. A seguir, temos a definição de uma base de Gröbner linear para $W$.

Definição. 2.4 Dizemos que um conjunto de vetores $\mathcal{G} \subset W$ é uma base de Gröbner linear para $W$ com respeito a boa ordem $>$ se

$$
\operatorname{Span}(\operatorname{Tip}(W))=\operatorname{Span}(\operatorname{Tip}(\mathcal{G}))
$$


Se $\mathcal{G}$ é uma base de Gröbner linear para $W$, então $\operatorname{Span}(\mathcal{G})=W$.

Pois, como $\mathcal{G} \subset W, \operatorname{Span}(\mathcal{G}) \subset W$. Suponha por absurdo que $W \not \subset$ $\operatorname{Span}(\mathcal{G})$, assim tome $w \in W \backslash \operatorname{Span}(\mathcal{G})$ com Tip $(w)$ minimal.

Como $\operatorname{Span}(\operatorname{Tip}(W))=\operatorname{Span}(\operatorname{Tip}(\mathcal{G}))$, existe $g \in \mathcal{G}$ tal que $\operatorname{Tip}(w)=$ $\operatorname{Tip}(g)$. Dessa forma, $w^{\prime}=w-(\operatorname{CTip}(w) / \operatorname{CTip}(g)) g \in \operatorname{Span}(\mathcal{G})$, pois $\operatorname{Tip}\left(w^{\prime}\right)<\operatorname{Tip}(w)$. Logo $w=w^{\prime}+(\operatorname{CTip}(w) / C T i p(g)) g \in \operatorname{Span}(\mathcal{G})$, que é um absurdo. Portanto, $\operatorname{Span}(\mathcal{G})=W$.

Definiçāo. 2.5 Seja $W$ um subespaço de $V$. Dizemos que um subconjunto $\mathcal{G}$ de $W$ é uma base de Gröbner linear reduzida para $W$ (com respeito a $>$ ) se:

(i) $\mathcal{G}$ é uma base de Gröbner linear para $W$;

(ii) S'e $g \in \mathcal{G}$, então $\operatorname{CTip}(g)=1$;

(iii) S'e $g$ e $g^{\prime}$ são elementos distintos de $\mathcal{G}$, então $\operatorname{Tip}(g) \neq \operatorname{Tip}\left(g^{\prime}\right)$;

(iv) Se $g \in \mathcal{G}$, então $g-\operatorname{Tip}(g) \in \operatorname{Span}(\operatorname{NonTip}(W))$.

Para garantir a existência de uma base de Gröbner linear reduzida temos o seguinte resultado:

Proposiçāo 2.6 Seja $V$ um espaço vetorial e > uma boa ordem na base $\mathcal{B}$ de V. Seja $W$ um subespaço de $V$. Então existe uma única base de Gröbner linear reduzida para $W$.

Prova. Seja $\mathcal{T}=\operatorname{Tip}(W)$ e defina $\mathcal{G}=\{t-N(t): t \in \mathcal{T}\}$. Pelo teorema 2.2, $t-N(t) \in W$. Agora, $\operatorname{Tip}(t-N(t))=t$ porque nenhum elemento ocorrendo em $N(t)$ pode ser Tip de um elemento em $W$. Logo $\mathcal{G}$ é uma base de Gröbner linear para $W$.

Ainda observando que para $g=t-N(t) \in \mathcal{G}$, Tip $(g)=t$, temos de forma direta as demais propriedades de uma base de Gröbner linear reduzida. 


\subsection{Bases de Gröbner para $\mathcal{K}$-Álgebras}

Agora, voltamos a nossa atenção para $\mathcal{K}$-álgebras, não necessariamente com unidade. Seja $\Lambda$ uma $\mathcal{K}$-álgebra e seja $\mathcal{B}$ uma $\mathcal{K}$-base de $\Lambda$. Assumimos que, para todo $b, b^{\prime} \in \mathcal{B}$ temos $b b^{\prime} \in \mathcal{B}$ ou $b b^{\prime}=0$. Tal $\mathcal{K}$-base é dita base multiplicativa de $\Lambda$.

Não queremos uma ordem arbitrária em $\mathcal{B}$, queremos uma ordem que preserve a estrutura multiplicativa de $\mathcal{B}$.

Definição. 2.7 Dizemos que uma boa ordem em $\mathcal{B}$ é admissivel, se satisfaz as seguintes condições, para todo $p, q, r, s \in \mathcal{B}$ :

(i) Se $p<q$ então $p r<q r$, se ambos são não nulos;

(ii) Se $p<q$ então $s p<s q$, se ambos são não nulos;

(iii) Se $p=$ sqr então $p \geq q$.

Como exemplo de uma $\mathcal{K}$-álgebra que possui uma $\mathcal{K}$-base multiplicativa com ordem admissível são as álgebras de caminhos $\mathcal{K} \mathcal{Q}$, tendo como base $\mathcal{B}$ o conjunto de todos os caminhos em $\mathcal{Q}$, incluindo os vértices, e a ordem é a comprimento-lexicográfica, isto é, dado às flechas e aos vértices uma ordenação arbitrária (ou seja, uma lexicografia), com os vértices menores que as flechas. Se $p$ e $q$ são caminhos de comprimento maiores que 1, dizemos que $p<q$ se o comprimento de $p$ for menor que o comprimento de $q$ ou se $p=p_{1} p_{2} \cdots p_{r}$ e $q=q_{1} q_{2} \cdots q_{r}$, com $p_{i}, q_{j}$ caminhos de comprimento 1 em $\mathcal{B}$, e para algum $1 \leq i \leq r, p_{j}=q_{j}$ se $j<i$ e $p_{i}<q_{i}$.

Dizemos que uma $\mathcal{K}$-álgebra $\Lambda$ possui a teoria de bases de Gröbner se $\Lambda$ possui uma base multiplicativa $\mathcal{B}$ com ordem admissível $>$ nessa base. $A$ partir desse ponto, assumimos que a $\mathcal{K}$-álgebra $\Lambda$ possui a teoria de bases de Gröbner. Seja $I$ um ideal bilateral em $\Lambda$. 
Definiçāo. 2.8 Dizemos que um conjunto $\mathcal{G} \subset I$ é uma base de Gröbner para I com respeito à ordem $>$, se

$$
\langle\operatorname{Tip}(\mathcal{G})\rangle=\langle\operatorname{Tip}(I)\rangle
$$

como ideais bilaterais.

Uma observação a se fazer é que aqui também é válido o teorema 2.2, ou seja, como espaços vetoriais

$$
\Lambda=I \oplus \operatorname{Span}(\operatorname{NonTip}(I))
$$

Em particular, todo $r \in \Lambda$ não nulo, pode ser escrito de forma única como $r=i_{r}+N(r)$, onde $i_{r} \in I$, e $N(r) \in \operatorname{Span}(N o n T i p(I))$. $N(r)$ é também chamado de forma normal de $r$.

Definição. 2.9 Sejam $b_{1}, b_{2} \in X \subset \Lambda$, dizemos que $b_{1}$ divide $b_{2}$ (em $X$ ) se existem $c, d \in X$ tais que $b_{2}=b_{1} d, b_{2}=c b_{1}$ ou $b_{2}=c b_{1} d$.

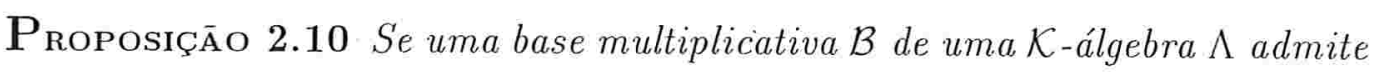
uma ordem admissível, então toda sequência infinita de $\mathcal{B}, b_{1}, b_{2}, \cdots$, tal que $b_{i+1}$ divide $b_{i}$ para todo $i \geq 1$ estabiliza, isto é, existe $N \in \mathbb{N}$ tal que $b_{N}=b_{N+k}$, para todo $k \in \mathbb{N}$.

Prova. Por definição, como $b_{i+1}$ divide $b_{i}$, então existem $c_{i}, d_{i} \in \mathcal{B}$, tais que $b_{i}=c_{i} b_{i+1} d_{i}$, para $i \geq 1$. Daí, $b_{i} \geq b_{i+1}$, ou seja, obtem-se uma cadeia descendente. Como a ordem dada é uma boa ordem, o resultado segue da proposição 1.12 .

Este resultado mostra que nem toda base multiplicativa possue uma ordem admissível. Como mostra o próximo exemplo: 
Exemplo. Seja $\mathcal{Q}$ o seguinte quiver:

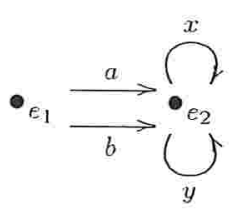

Seja o ideal $I=<a x^{n}-b, a-b y^{m}: m, n \in \mathbb{N}^{*}>$. Uma base multiplicativa $\mathcal{B}$ de $\Lambda=\mathcal{K} \mathcal{Q}$ é o conjunto de todos os caminhos de $\mathcal{Q}$. Note que, em $\Lambda / I$, temos $\overline{a x^{n}}=\overline{b y^{m} x^{n}}=\bar{b}$ e $\overline{b y^{m}}=\overline{a x^{n} y^{m}}=\bar{a}$ para todo $n, m \in \mathbb{N}$, assim podemos considerar em $\Lambda / I$ a seguinte base

$$
\overline{\mathcal{B}}=\mathcal{B} \backslash\left\{b x^{n}, a y^{m}, a x^{n}, b y^{m}, a x^{n} y^{m}, b y^{m} x^{n}: m, n \in \mathbb{N}^{*}\right\} .
$$

Note que $\overline{\mathcal{B}}$ contém uma base de $\mathcal{K}\langle x, y\rangle$. Note também que, $\bar{a} \bar{x}=\bar{b}$ o que implica em dizer que $\bar{a}$ divide $\bar{b}$. Por outro lado, $\bar{b} \bar{y}=\bar{a}$, logo, $\bar{b}$ divide $\bar{a}$. Assim, temos uma sequência descendente infinita $\bar{a}, \bar{b}, \bar{a}, \bar{b}, \cdots$ que não estabiliza, portanto $\overline{\mathcal{B}}$ não possui uma ordem admissivel.

Dada uma base $\mathcal{B}$, chamaremos de monômios seus elementos. Diremos que um ideal $I$ em $\Lambda$ é um ideal monomial se ele pode ser gerado por elementos de $\mathcal{B}$.

Proposiçāo 2.11 Seja $\Lambda$ uma $\mathcal{K}$-álgebra com base multiplicativa $\mathcal{B}$ que admite uma ordem admissivel $>$. Se I é um ideal monomial em $\Lambda$, então I tem um único conjunto gerador monomial minimal, em relação à divisão.

Prova. Seja $A$ o conjunto de todos os monômios em $I$. Seja

$$
M=\{p \in A: \text { se } q \in A \text { divide } p, \text { então } q=p\}
$$

Pela proposição anterior temos que $M$ é não vazio.

Seja $\mathcal{C}$ um conjunto de monômios que geram $I$. Se $b \in \mathcal{C}$, então, para algum $m \in M, m$ divide $b$. Assim, $\mathcal{C} \subset\langle M\rangle$. Logo, $I \subset\langle M\rangle$. 
Agora, seja $M^{\prime}$ um outro conjunto de monômios geradores de $I$, então todo $m \in M$ é divisível por algum $m^{\prime} \in M^{\prime}$. Mas, pela definição de $M$, $m^{\prime}=m$. Logo, $M \subset M^{\prime}$.

Note que o conjunto minimal de geradores $M$ não depende de qualquer ordem admissível particular. A existência de uma ordem admissível é necessária apenas para mostrar que $M$ é não vazio.

Observe ainda que para todo ideal $I$ de $\Lambda$, se $\mathcal{G}$ é uma base de Gröbner de $I$, então Tip $(\mathcal{G})$ contém o conjunto gerador monomial minimal de $\langle$ Tip $(I)\rangle$.

Seja $\mathcal{T}$ o conjunto gerador monomial minimal de $\langle\operatorname{Tip}(I)\rangle$ (dada uma ordem admissível $>$ ).

Definiçāo. 2.12 A base de Gröbner reduzida para I, com respeito à ordem $>e^{\prime}$

$$
\mathcal{G}=\{t-N(t): t \in \mathcal{T}\}
$$

Proposição 2.13 Seja > uma ordem admissível na base multiplicativa $\mathcal{B}$ da $\mathcal{K}$-álgebra $\Lambda$. Sejam I um ideal em $\Lambda$ e $\mathcal{G}$ uma base de Gröbner reduzida para I. Então:

(i) $\mathcal{G}$ é uma base de Gröbner de I;

(ii) Se $g \in \mathcal{G}$, entẫo $\operatorname{CTip}(g)=1$;

(iii) Se $g \in \mathcal{G}$, então $g-\operatorname{Tip}(g) \in \operatorname{Span}($ NonTip $(I))$;

(iv) $\operatorname{Tip}(\mathcal{G})$ é o conjunto gerador monomial minimal para $\langle$ Tip $(I)\rangle$

\subsection{Algoritmo da Divisão}

Nesta seção, fixamos um corpo $\mathcal{K}$, uma $\mathcal{K}$-álgebra $\Lambda$ com base multiplicativa $\mathcal{B}$ e uma ordem admissivel $>$ nessa base. 
Dados $x, y \in \Lambda$, temos visto como podemos dividir $y$ por $x$ em $\Lambda$, definição 2.9. Apresentamos agora o algoritmo da divisão, onde mostraremos como dividir um elemento $y \in \Lambda$ por um dado conjunto $X$. Enfatizamos aqui que a ordenação no conjunto $X$ afeta no resultado do algoritmo da divisão, como veremos mais tarde.

Seja $X=\left\{x_{1}, \cdots, x_{n}\right\}$ um conjunto ordenado de elementos de $\Lambda$ e $y \in \Lambda$.

$\mathrm{O}$ algoritmo nos fornecerá inteiros não negativos $m_{1}, m_{2}, \cdots, m_{n}$ e elementos $u_{i j}, v_{i j} \in \Lambda 1 \leq i \leq n$ e $1 \leq j \leq m_{i}$ tais que:

(i) $y=\sum_{i=1}^{n}\left(\sum_{j=1}^{m_{i}} u_{i j} x_{i} v_{i j}\right)+r$;

(ii) $\operatorname{Tip}(y) \geq \operatorname{Tip}\left(u_{i j} x_{i} v_{i j}\right) \forall i, j$;

(iii) Para cada $b \in \mathcal{B}$ tal que $b$ ocorra em $r$, Tip $\left(x_{i}\right)$ não divide $b, 1 \leq i \leq n$.

Chamaremos $r$ de resto da divisão de $y$ por $X$ e denotaremos por $y \Rightarrow_{X} r$. Apresentamos abaixo o algoritmo em pseudo-código:

\section{O Algoritmo da Divisão}

INPUT: $x_{1}, \cdots, x_{n}$ (ordenado), $y$

OUTPUT: $m_{1}, \cdots, m_{n}, u_{i j}, v_{i j}, r$

INICIALIZE: $m_{1}:=0, \cdots, m_{n}:=0, r:=0, z:=y$, INÍCIO:

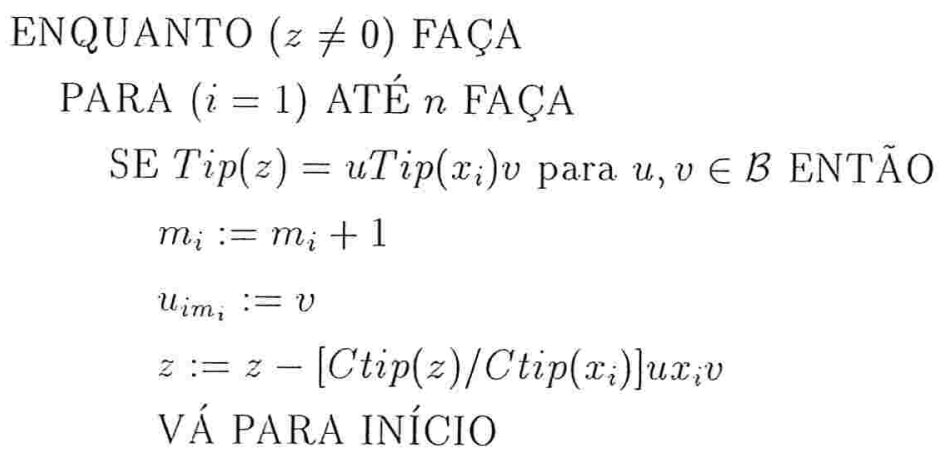




$$
\begin{aligned}
& r:=r+C \operatorname{tip}(z) \operatorname{Tip}(z) \\
& z:=z-\operatorname{Ctip}(z) \operatorname{Tip}(z)
\end{aligned}
$$

Antes de prosseguirmos, veremos um exemplo em que a ordem do conjunto $X$ dada inicialmente influi no resto $r$ obtido através do algoritmo da divisão:

Exемplo. Seja $\mathcal{Q}$ o quiver

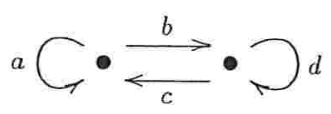

Seja a álgebra de caminhos $\mathcal{K} \mathcal{Q}$ com base multiplicativa $\mathcal{B}$, formada por todos os possíveis caminhos do quiver, com a ordem comprimento-lexicográfica, onde a lexicografia é dada por $a<b<c<d$.

Tomemos $X=\left\{f_{1}=c a b-a b, f_{2}=a b d c-d c\right\}$ e $y=b c a b d c$.

Iniciando o algoritmo, vemos que $z=b c a b d c=b \operatorname{Tip}\left(f_{1}\right) d c$.

Assim, $u_{11}=b, v_{11}=d c$ e $z:=b c a b d c-u_{11} f_{1} v_{11}=-b a b d c$. Agora, como $z=b \operatorname{Tip}\left(f_{2}\right), u_{12}:=b$ e $z:=-b a b d c-[\operatorname{CTip}(z)] u_{12} f_{2}=b d c$.

Note que, nem Tip $\left(f_{1}\right)$, nem Tip $\left(f_{2}\right)$ divide $\operatorname{Tip}(z)=b d c, \operatorname{logo}$ o resto $r=b d c$, e agora $z$ toma o valor zero, parando assim o algoritmo, retornando:

$$
b c a b d c=b f_{1} d c+b f_{2}-b d c .
$$

No entanto, se invertermos a ordenação em $X$, ou seja, se iniciarmos o algoritmo tomando primeiramente o elemento $f_{2}$, veremos que o algoritmo nos retornará

$$
b c a b d c=b c f_{2},
$$

pois $(b c)(d c)=0$. Note que o resto nesse caso é $r=0$.

Como podemos perceber, a ordem influencia no resto da divisão. No entanto, se $X$ for uma base de Gröbner, então o resto da divisão independe da ordenação em $X$, como podemos ver no próximo resultado. 
$\mathrm{P}_{\text {Roposiçāo }} 2.14$ Seja $\mathcal{G}$ uma base de Gröbner de um ideal I de $\Lambda$. S'eja $y \in \Lambda$ e assuma que $X=\{g \in \mathcal{G}: \operatorname{Tip}(g) \leq \operatorname{Tip}(y)\}$ seja finito. Se $y \Rightarrow_{X} r$, então $r$ independe da ordem dos elementos de $X$. Na verdade $r=N(y)$.

Prova. Considere $y \Rightarrow_{X} r$. Então, como $\operatorname{Tip}(r) \leq \operatorname{Tip}(y)$, temos que para cada $g \in \mathcal{G}, \operatorname{Tip}(g)$ não divide nenhum elemento da base ocorrendo em $r$.

Assim $r \in \operatorname{Span}(\operatorname{Nontip}(I))$. Agora, $y=\sum_{i} \sum_{j} u_{i j} g_{i} v_{i j}+r$. Mas, pelo teorema 2.2, $y=i_{y}+N(y) \operatorname{com} i_{y} \in I$ e $N(y) \in \operatorname{Span}(N o n t i p(I))$, únicos.

Assim, como $\sum_{i} \sum_{j} u_{i j} g_{i} v_{i j} \in I$, temos $r \in \operatorname{Span}(\operatorname{Nontip}(I))$, e portanto $r=N(y)$.

Corolário 2.15 Se $\mathcal{G}$ é uma base de Gröbner de um ideal I em $\Lambda$ tal que para cada $b \in \mathcal{B}$ exista somente um número finito de elementos $g \in \mathcal{G}$ com $\operatorname{Tip}(g) \leq b$, então existe um algoritmo para encontrar a forma normal de elementos de $\Lambda$.

Note que agora temos também um método para encontrar a base de Gröbner reduzida uma vez encontrada uma base de Gröbner com um número finito de termos com um dado Tip. Note que, com essa hipótese, não é necessário que a base de Gröbner seja finita. Pois, para cada $x \in \Lambda$, temos apenas um número finito de elementos $g \in \mathcal{G}$ tais que $\operatorname{Tip}(g) \leq \operatorname{Tip}(x)$. Viabilizando assim o uso do algoritmo da divisão.

Este é um algoritmo, isto é, o processo termina, se $I_{M O N}=\langle$ Tip $(I)\rangle$ tem um conjunto de monômios gerador finito.

O método procede da seguinte forma:

(i) Dada uma base de Gröbner $\mathcal{G}$ tal que somente um número finito de termos possui um dado Tip. 
(ii) Encontre o conjunto gerador monomial minimal $\mathcal{T}$ de $I_{M O N}$.

(iii) Para cada $t \in \mathcal{T}$, usando o algoritmo da divisão, calcule $t \Rightarrow_{\mathcal{G}} N(t)$.

(iv) A base de Gröbner reduzida é $\{t-N(t): t \in \mathcal{T}\}$.

\subsection{Relações de Sobreposição}

Definiçāo. 2.16 Sejam $f, g \in \Lambda$ e suponha que existam $b, c \in \mathcal{B}$ tais que:

(i) $\operatorname{Tip}(f) c=b \operatorname{Tip}(g)$

(ii) Tip(f) não divide $b$ e Tip $(g)$ não divide $c$.

Então a relação de sobreposição de $f$ e g por b e c é dada por:

$$
o(f, g, b, c)=(1 / C \operatorname{Tip}(f)) f c-(1 / C \operatorname{Tip}(g)) b g
$$

Exemplo. Seja a álgebra livre $\mathcal{K}\langle x, y, z\rangle$, com $\mathcal{K}$-base gerada por $1, x, y, z$ e ordem comprimento lexicográfica (que é uma ordem admissível), onde a lexicografia é dada por $1<x<y<z$.

Sejam $f=z x y y z-x y z$ e $g=x y x-x y$. Como Tip $(f)=z x y y x$ e $\operatorname{Tip}(g)=x y x$, note que

$$
\operatorname{Tip}(f) y x=z x y y \operatorname{Tip}(g)
$$

e Tip $(f)$ não divide $z x y y$ e Tip $(g)$ não divide $y x$. Portanto, temos aqui uma relação de sobreposição

$$
\begin{aligned}
o(f, g, z x y y, y x) & =f y x-z x y y g=(z x y y x-x y z) y x-z x y y(x y x-x y) \\
& =z x y y x y x-x y z y x-z x y y x y x+z x y y x y \\
& =\text { zxyyxy-xyzyx}
\end{aligned}
$$


Temos ainda que, Tip $(g) y x=x y$ Tip $(g)$ e que Tip $(g)$ não divide $x y$ e não divide $y x$, assim temos aqui mais uma relação de sobreposição de $g$ sobre ele mesmo:

$$
\begin{aligned}
o(g, g, x y, y x) & =g y x-x y g=(x y x-x y) y x-x y(x y x-x y) \\
& =x y x y x-x y y x-x y x y x+x y x y \\
& =-x y y x+x y x y
\end{aligned}
$$

Note que Tip $(o(f, g, b, c))<\operatorname{Tip}(f) c=b \operatorname{Tip}(g)$.

Suponha que $X=\left\{x_{1}, \cdots, x_{n}\right\} \subset \Lambda$. Seja $I$ o ideal gerado por $X$.

Se Tip $\left(x_{i}\right)=u \operatorname{Tip}\left(x_{j}\right) v$ para algum $u, v \in \mathcal{B}$, então fazendo

$$
X^{\prime}=\left\{x_{1}, \cdots, x_{i-1}, x_{i}-\left[\operatorname{CTip}\left(x_{i}\right) / \operatorname{CTip}\left(x_{j}\right)\right] u x_{j} v, x_{i+1}, \cdots, x_{n}\right\}
$$

temos que $X^{\prime}$ é também gerador de $I$.

Continuando o processo, obtemos um conjunto finito $Y$ gerador de $I$ tal que Tip $\left(y_{i}\right)$ não divide Tip $\left(y_{j}\right)$, para $y_{i}, y_{j} \in Y \operatorname{com} i \neq j$.

Este é um processo finito pela boa ordem assumida. Assim podemos assumir que não há divisões de Tip's em um conjunto gerador de um ideal.

Definiçāo. 2.17 Dizemos que um conjunto de elementos $X$ é Tip reduzido se para elementos distintos $x, y \in X$, Tip $(x)$ não divide Tip $(y)$.

Definição. 2.18 Dizemos que um elemento $f=\sum_{i=1}^{n} \alpha_{i} b_{i}$, com $b_{i} \in \mathcal{B} e$ $\alpha_{i} \in \mathcal{K} \backslash\{0\}$ para $1 \leq i \leq n$ é (à esquerda) uniforme se para cada $c \in \mathcal{B}$, ou $c b_{i}=0$ para $1 \leq i \leq n$, ou $c b_{i} \neq 0$ para $1 \leq i \leq n$.

Se um dado conjunto possui apenas elementos uniformes dizemos então que esse conjunto é uniforme.

Definiçāo. 2.19 Seja $\Lambda$ uma $\mathcal{K}$-álgebra com base multiplicativa $\mathcal{B}$ e uma ordem admissivel $>$ em $B$. Seja $\mathcal{C}$ o conjunto formado pelos elementos indecomponiveis de $\mathcal{B}$. Para todo $b \in \mathcal{B}$ definimos o comprimento de $b, \ell(b)$, como sendo o menor inteiro $n$ tal que $b=c_{1} c_{2} \cdots c_{n}$, onde $c_{i} \in \mathcal{C}$ com $1 \leq i \leq n$. 
Cabe observar que o conjunto $\mathcal{C}$ pode ser infinito, no entanto, $\ell(b)$ é sempre finito, pois se $b=c_{1} c_{2} c_{3} c_{3} \cdots$, teríamos uma sequência infinita, $b, c_{2} c_{3} c_{4} \cdots, c_{3} c_{4} \cdots, \cdots$, com $b \geq c_{2} c_{3} c_{4} \cdots \geq c_{3} c_{4} \cdots \geq \cdots$, contradizendo a. hipótese da boa ordenação em $\mathcal{B}$.

Podemos notar ainda que, o comprimento de um elemento depende da escolha da base multiplicativa $\mathcal{B}$. O resultado abaixo, descrito em [10], é uma versão do Lema do Diamante (veja [5], [7]) e será muito importante para a construção de uma base de Gröbner mais adiante.

Teorema 2.20 Seja $\Lambda$ uma $\mathcal{K}$-álgebra com base multiplicativa $\mathcal{B}$ e ordem admissivel $>$. Suponha que $\mathcal{G}$ é um conjunto uniforme, Tip reduzido de $\Lambda$. Suponha que para toda relação de sobreposição

$$
o\left(g_{1}, g_{2}, p, q\right) \Rightarrow_{\mathcal{G}} 0
$$

com $g_{1}, g_{2} \in \mathcal{G}$. Então $\mathcal{G}$ é uma base de Gröbner para $\langle\mathcal{G}\rangle$.

Prova. Assuma que $\mathcal{G}$ tem a propriedade de que toda relação de sobreposição tem resto zero sobre a divisão por $\mathcal{G}$. Seja $x \in\langle\mathcal{G}\rangle$ e assuma que Tip $(x)$ não é divisível pelo Tip de nenhum elemento de $\mathcal{G}$, veremos que isto nos levará a uma contradição. Sem perda de generalidade, vamos assumir que $x$ é uniforme. Como $\mathcal{G}$ é um conjunto gerador de $\langle\mathcal{G}\rangle$, podemos escrever:

$$
x=\sum_{i, j} \alpha_{i j} p_{i j} g_{i} q_{i j}
$$

onde $g_{i} \in \mathcal{G}, p_{i j}, q_{i j} \in \mathcal{B}$ e $\alpha_{i j} \in \mathcal{K}$. Considere todas as formas de se escrever $x$. Seja. $\bar{p}$ o maior elemento (com respeito à ordem dada) da base $\mathcal{B}$ ocorrendo do lado direito de $(*)$. Como estamos assumindo que Tip $(x)$ não é divisível pelo Tip de nenhum elemento de $\mathcal{G}$, temos pela uniformidade de $x$ que $\bar{p}$ é maior que Tip $(x)$ na ordem $>$. Logo, os $\bar{p}$ 's se cancelam.

Considerando todas formas de escrever $x$ como em $(*)$, escolhemos aquela em que $\bar{p}$ seja o menor possível e tenha o menor número de ocorrências do lado direito de $(*)$. 
Como $\bar{p}$ não ocorre do lado esquerdo de (*), ele deve aparecer em pelo menos dois somandos do lado direito de $(*)$. Logo, existem $i, j, i^{\prime}, j^{\prime}$ tais que

$$
\bar{p}=p_{i j} \operatorname{Tip}\left(g_{i}\right) q_{i j}=p_{i^{\prime} j^{\prime}} \operatorname{Tip}\left(g_{i^{\prime}}\right) q_{i^{\prime} j^{\prime}} .
$$

Para simplificar a notação escreveremos $p=p_{i j}, g=g_{i}, q=q_{i j}, p^{\prime}=$ $p_{i^{\prime} j^{\prime}}, g^{\prime}=g_{i^{\prime}}$ e $q^{\prime}=q_{i^{\prime} j^{\prime}}$.

Caso 1: $\ell(p)<\ell\left(p^{\prime}\right)$.

Caso 1.1: $\ell(q)<\ell\left(q^{\prime}\right)$. Então Tip $\left(g^{\prime}\right)$ é divisível por Tip $(g)$ e assim Tip $(g)$ divide Tip $\left(g^{\prime}\right)$ contradizendo a hipótese de Tip redução.

Caso 1.2: $\ell(q) \geq \ell\left(q^{\prime}\right)$. Consideremos duas possibilidades:

Caso 1.2.1: $\ell\left(p^{\prime}\right) \geq \ell(p T i p(g))$.

Então não existe relação de sobreposição entre Tip $(g)$ e Tip $\left(g^{\prime}\right)$ em $\bar{p}$.

Pela escolha dos comprimentos, segue que existe um caminho $q^{\prime \prime}$ tal que $\bar{p}=p \operatorname{Tip}(g) q^{\prime \prime} \operatorname{Tip}\left(g^{\prime}\right) q^{\prime}$.

Agora, se $g=\sum \alpha_{r} p_{r}+\alpha \operatorname{Tip}(g)$ e $g^{\prime}=\sum \beta_{s} p_{s}^{\prime}+\beta \operatorname{Tip}\left(g^{\prime}\right)$, então:

$$
\begin{aligned}
p g q & =p g q^{\prime \prime}(1 / \beta) g^{\prime} q^{\prime}-p g q^{\prime \prime}(1 / \beta)\left(g^{\prime}-\operatorname{Tip}\left(g^{\prime}\right)\right) q^{\prime} \\
& =(\alpha / \beta) p \operatorname{Tip}(g) q^{\prime \prime} g^{\prime} q^{\prime}+\sum\left(\alpha_{r} / \beta\right) p p_{r} q^{\prime \prime} g^{\prime} q^{\prime}-\sum\left(\beta_{s} / \beta\right) p g q^{\prime \prime} p_{s}^{\prime} q^{\prime} .
\end{aligned}
$$

Logo, escrito $p g q$ desta forma, podemos combinar seu Tip com o Tip de $p^{\prime} g^{\prime} q^{\prime}$ e assim, diminue o número de ocorrências de $\bar{p}$, que contradiz a minimalidade assumida.

Caso 1.2.2: $\ell\left(p^{\prime}\right)<\ell(p$ Tip $(g))$

Então existe uma relação de sobreposição de Tip $(g)$ e Tip $\left(g^{\prime}\right)$ em $\bar{p}$. Digamos Tip $(g) r=\operatorname{sTip}\left(g^{\prime}\right)$. Logo, $\bar{p}=p$ Tip $(g) r q^{\prime}=p s$ Tip $\left(g^{\prime}\right) q^{\prime}$. Então

$$
p g q=C \operatorname{Tip}(g) p o\left(g, g^{\prime}, s, r\right) q^{\prime}+\left(\operatorname{CTip}(g) / C T i p\left(g^{\prime}\right)\right) p^{\prime} g^{\prime} q^{\prime} .
$$

Como $o\left(g, g^{\prime}, s, r\right) \Rightarrow_{\mathcal{G}} 0$, então

$$
o\left(g, g^{\prime}, s, r\right)=\sum_{i=1}^{n} \sum_{j=1}^{m_{i}} u_{i j} g_{i} v_{i j},
$$


onde Tip $\left(u_{i j} g_{i} v_{i, j}\right)$ é menor que Tip $(g) r=\operatorname{sTip}\left(g^{\prime}\right)$, para todo $i, j$.

Desse modo,

$$
p g q+p^{\prime} g^{\prime} q^{\prime}=p g r q^{\prime}-\left(\frac{C \operatorname{Tip}(g)}{\operatorname{CTip}\left(g^{\prime}\right)}\right) p s g^{\prime} q^{\prime}+\left(\frac{\operatorname{CTip}(g)}{\operatorname{CTip}\left(g^{\prime}\right)}\right) p^{\prime} g^{\prime} q^{\prime}+p^{\prime} g^{\prime} q^{\prime} .
$$

De acordo com as relações assumidas acima, temos que $\bar{p}$ ocorre em $p g r q^{\prime}$, $p s g^{\prime} q^{\prime}$ e $p^{\prime} g^{\prime} q^{\prime}$, que contradiz a minimalidade assumida.

Caso $2: \ell(p)=\ell\left(p^{\prime}\right)$

Então Tip $(g)$ divide $\operatorname{Tip}\left(g^{\prime}\right)$, ou vice-versa, que contradiz a hipótese de Tip reduzido em $\mathcal{G}$.

Caso $3: \ell(p)>\ell\left(p^{\prime}\right)$

Como no Caso 1.

Uma aplicação desse último resultado está no próximo algoritmo, que é o análogo não comutativo do algoritmo de Buchberger [6] para construção de bases de Gröbner.

Dado $I=\left\langle f_{1}, \cdots, f_{n}\right\rangle, f_{i} \in \Lambda$, uniformes, para todo $1 \leq i \leq n$ e Tip reduzidos, o algoritmo produz uma sequência, possivelmente infinita, de elementos Tip reduzidos e uniformes. Além disso, a sequência $\mathcal{G}$ obtida, é um conjunto gerador de $I$ onde toda relação de sobreposição se reduz a zero sobre $\mathcal{G}$. Portanto, pelo teorema anterior, $\mathcal{G}$ é uma base de Gröbner para $I$. Apresentamos abaixo o algoritmo em pseudo-código.

\section{Construção de base de Gröbner}

INPUT: $f_{1}, \cdots, f_{n}$

OUTPUT: $g_{1}, g_{2}, g_{3}, \cdots$

PARA $i=1$ ATÉ $n$ FAÇA

$$
\begin{gathered}
g_{i}:=f_{i} \\
\mathcal{G}:=\left\{g_{1}, \cdots, g_{n}\right\} \\
\text { Count }:=n
\end{gathered}
$$




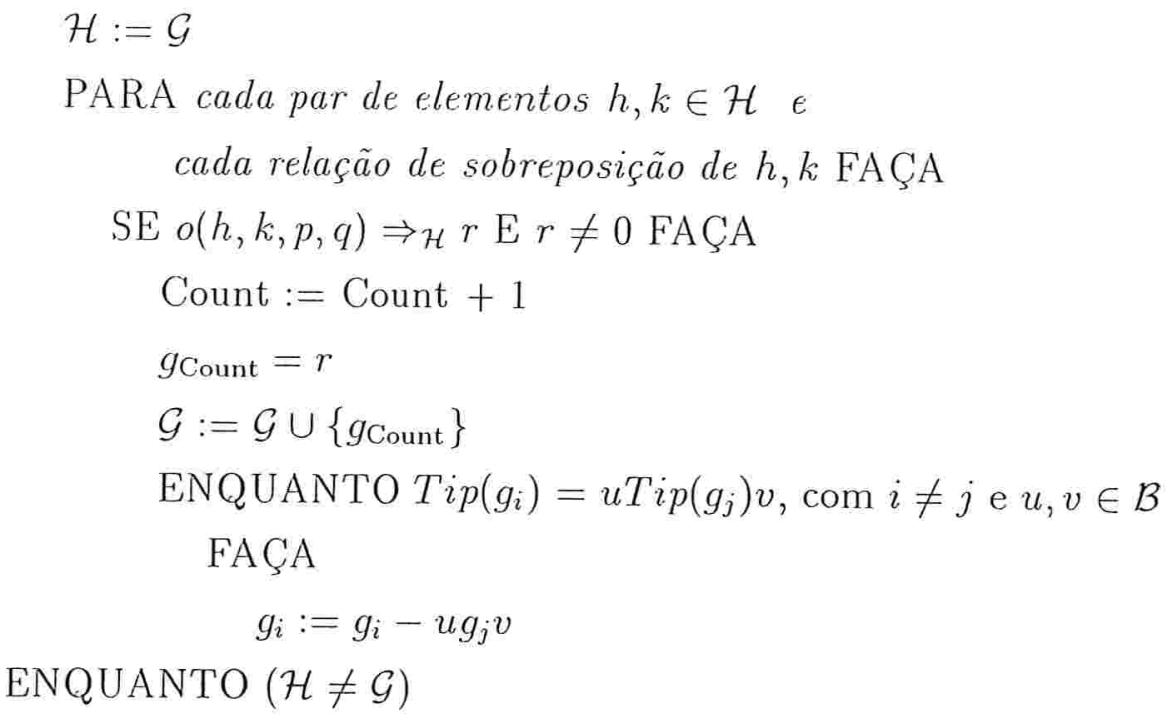

Lema 2.21 Utilizando a notação usada no algoritmo. Se $b \in \mathcal{B}$ é um gerador monomial minimal de $I_{M O N}$, então para algum $m$ inteiro, $\operatorname{Tip}\left(g_{m}\right)=b$ 】 Proposição 2.22 Se $I_{\text {MON }}$ tem um conjunto finito de geradores monomiais, então o algoritmo acima termina em um número finito de passos e fornece uma base de Gröbner finita.

Prova. Seja $\mathcal{T}=\left\{t_{1}, \cdots, t_{s}\right\}$ o conjunto gerador monomial minimal de $I_{M O N}$.

Assim, pelo lema anterior, $\mathcal{T} \subset\left\{\operatorname{Tip}\left(g_{1}\right), \cdots, \operatorname{Tip}\left(g_{N}\right)\right\}$, para $N$ suficientemente grande. $\operatorname{Logo} \operatorname{Tip}\left(\left\{g_{1}, \cdots, g_{N}\right\}\right)$ gera $I_{M O N}$ sendo portanto uma base de Gröbner para $I$.

Como toda relação de sobreposição está em $I, o\left(g_{i}, g_{j}, p, q\right) \Rightarrow_{\left\{g_{1}, \cdots, g_{N}\right\}} 0$. Logo o algoritmo termina em um número finito de passos.

Exemplo. Nem todo ideal I possui uma base de Gröbner finita, mesmo que $I$ seja finitamente gerado, como podemos ver abaixo: 
Seja $\mathcal{Q}$ o quiver:

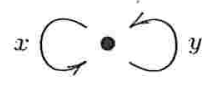

Seja $I=\langle f=x y x-x y\rangle$ ideal bilateral em $\mathcal{K} \mathcal{Q}$, cuja $\mathcal{K}$-base é o conjunto de todos os caminhos de $\mathcal{Q}$ e a ordem é o comprimento lexicográfica, com a lexicografia dada por $1<x<y$.

Seja $\mathcal{G}_{0}=\{f\}$. Note que, $o_{1}=o(f, f, x y, y x)=x y^{2} x+x y x y$ e fazendo a. divisão por $\mathcal{G}_{0}$, temos que $o_{1} \Rightarrow \mathcal{G}_{0} g_{1}=-x y^{2} x+x y^{2}$ e Tip $(f)$ não divide Tip $\left(g_{1}\right)$. Calculamos então $o_{2}=o\left(f, g_{1}, x y, y^{2} x\right)=-x y^{3} x+x y x y^{2}$, fazendo a divisão por $\mathcal{G}_{1}=\mathcal{G}_{0} \cup\left\{g_{1}\right\}$, obtemos $o_{2} \Rightarrow_{\mathcal{G}_{1}} g_{2}=-x y^{3} x+x y^{3}$, e observe que nem $\operatorname{Tip}(f)=x y x$ e nem $\operatorname{Tip}\left(g_{1}\right)=x y^{2} x$ dividem $\operatorname{Tip}\left(g_{2}\right)=x y^{3} x$, acrescentamos então $g_{2}$ a $\mathcal{G}_{1}$, fazendo $\mathcal{G}_{2}=\mathcal{G}_{1} \cup\left\{g_{2}\right\}$. Continuando o processo, temos que $o_{n}=o\left(f, g_{n-1}, x y, y^{n} x\right) \Rightarrow_{g_{n-1}} g_{n}=-x y^{n+1} x+x y^{n+1}$, sendo que nenhum dos Tip's anteriores divide Tip $\left(g_{n}\right)$. Podemos concluir então que, se utilizarmos o processo de construção de bases de Gröbner descrito acima encontraremos em $\mathcal{G}$ o conjunto de elementos cujo Tip's são da forma $x y^{n} x$, com $n \in \mathbb{N}$, e teremos portanto uma base de Gröbner infinita, pois para cada elemento em $\operatorname{Tip}(\mathcal{G})$ temos um elemento em $\mathcal{G}$.

\section{$2.5 \mathcal{K}$-álgebras de Dimensão Finita}

Nesta seção voltaremos nossa atenção para as $\mathcal{K}$-álgebras de dimensão finita da forma $\Lambda / I$, onde $\Lambda$ é uma $\mathcal{K}$-álgebra finitamente gerada, com uma base multiplicativa e uma ordem admissível nessa base, e $I$ é um ideal bilateral de $\Lambda$. O que mais podemos dizer a respeito dessas álgebras?

Antes, lembremos que $I_{M O N}=\langle\operatorname{Tip}(I)\rangle$.

Lema 2.23 Seja $\Lambda$ uma $\mathcal{K}$-álgebra finitamente gerada e $\mathcal{B}$ uma $\mathcal{K}$-base multiplicativa de $\Lambda$. Então $\mathcal{B} \cup\{0\}$ é finitamente gerada como semigrupo. 
Prova. Seja $X=\left\{x_{1}, x_{2}, \cdots, x_{n}\right\}$ um conjunto gerador de $\Lambda$ como $\mathcal{K}$ álgebra. Para cada $x_{i} \in X, 1 \leq i \leq n$, temos $x_{i}=\sum_{j} \alpha_{i j} b_{i j}$, com $b_{i j} \in \mathcal{B}$ e $\alpha_{i j} \in \mathcal{K}$. Seja $\overline{\mathcal{B}}=\bigcup_{i=1}^{n} \operatorname{supp}\left(x_{i}\right)$, é fácil ver que $\overline{\mathcal{B}}$ é finito.

$\overline{\mathcal{B}}$ gera $\mathcal{B} \cup\{0\}$ como semigrupo. Suponha, por contradição, que $\overline{\mathcal{B}}$ não gera $\mathcal{B} \cup\{0\}$ como semigrupo, daí existe $b \in \mathcal{B}$ tal que $b$ não pode ser escrito como produto de elementos de $\overline{\mathcal{B}}$. Como $X$ é gerador de $\Lambda$, temos

$$
\begin{aligned}
b & =\sum_{i} \beta_{i} x_{i_{1}}^{t_{i_{1}}} x_{i_{2}}^{t_{i_{2}}} \cdots x_{i_{r_{i}}}^{t_{i_{r_{i}}}} \\
& =\sum_{i} \beta_{i}\left(\sum_{j_{1}} \alpha_{i_{1} j_{1}} b_{i_{1} j_{1}}\right)^{t_{i_{1}}}\left(\sum_{j_{2}} \alpha_{i_{2} j_{2}} b_{i_{2} j_{2}}\right)^{t_{i_{2}}} \cdots\left(\sum_{j_{r_{i}}} \alpha_{i_{r_{i}} j_{r_{i}}} b_{i_{r_{i}} j_{r_{i}}}\right)^{t_{i_{r_{i}}}}
\end{aligned}
$$

Note que, do lado direito da equação temos uma combinação linear de produtos de elementos de $\overline{\mathcal{B}}$, que por sua vez são elementos de $\mathcal{B} \cup\{0\}$.

Como $b$ não é produto de elementos de $\overline{\mathcal{B}}$, então $b$ não aparece do lado direito da equação. Assim, temos que $b$ é uma combinação linear de elementos de $\mathcal{B} \backslash\{b\}$, uma contradição, pois $\mathcal{B}$ é uma base.

Portanto, $\overline{\mathcal{B}}$ gera $\mathcal{B} \cup\{0\}$ como semigrupo.

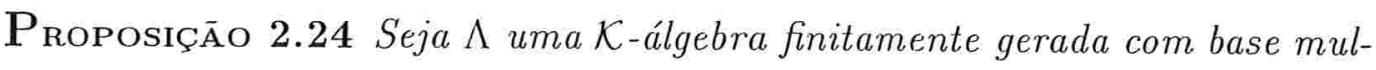
tiplicativa $\mathcal{B}$ e ordem admissivel $>$ nessa base. Suponha que $I$ é um ideal tal que $\operatorname{dim}_{\mathcal{K}}(\Lambda / I)=N$. Então $I_{M O N}$ tem um conjunto finito de geradores monomiais.

Prova. Como $\Lambda / I$ é isomorfo a $\operatorname{Span}(\operatorname{NonTip}(I))$, como espaço vetorial, segue que $\operatorname{NonTip}(I)$ é um conjunto finito, pois é uma $\mathcal{K}$-base de $\operatorname{Span}(\operatorname{NonTip}(I))$.

Como $\Lambda$ é finitamente gerada como $\mathcal{K}$-álgebra, temos que $\mathcal{B} \cup\{0\}$ é finitamente gerado como semigrupo (lema acima). Seja $X=\left\{b_{1}, \cdots, b_{k}\right\}$ gerador de $\mathcal{B} \cup\{0\}$, como semigrupo. Mostraremos que

$$
\mathcal{D}=(\{b c: b \in X \text { e } c \in N o n \operatorname{Tip}(I)\} \cap \operatorname{Tip}(I)) \cup(X \cap \operatorname{Tip}(I))
$$


contém o conjunto gerador de $I_{M O N}$.

Suponha que $t$ pertence ao conjunto gerador monomial minimal de $I_{M O N}$. Se $t \in X$, nada temos a fazer. Suponha que $t \notin X$, então $t=b_{i_{1}} b_{i_{2}} \cdots b_{i_{s}}$, com $b_{i j} \in X$ para todo $1 \leq j \leq s$. Pela minimalidade de $t$, temos que $b_{i_{2}} \cdots b_{i_{s}} \notin$ Tip $(I)$. Logo $b_{i_{2}} \cdots b_{i_{s}} \in N$ onTip $(I)$.

Portanto, fazendo $c=b_{i_{2}} \cdots b_{i_{s}}$, temos que $t=b_{i_{1}} c$. Como queríamos.

Corolário 2.25 Seja $\Lambda$ uma $\mathcal{K}$-álgebra finitamente gerada com base multiplicativa $\mathcal{B}$ e uma ordem admissivel $>$ em $\mathcal{B}$. Suponha que I é um ideal de $\Lambda$ gerado por elementos uniformes e $\Lambda / I$ de dimensão finita.

Então I tem uma base de Gröbner finita uniforme com respeito $a>e$ pode ser calculado em um número finito de passos pelo algoritmo anterior. 


\section{Capítulo 3}

\section{Bases de Gröbner e Álgebra de Caminhos}

\subsection{Por que Álgebras de Caminhos?}

Nesta seção vamos ver um pouco mais sobre a relação entre as álgebras que admitem uma base multiplicativa com uma ordem admissível e as álgebras de caminhos.

E também por que o uso dessas álgebras é necessário para se estudar a teoria de bases de Gröbner. Na verdade, veremos que toda álgebra com unidade que admite uma base multiplicativa com uma ordem admissível é quociente de uma álgebra de caminhos. Os resultados encontrados nessa seção foram demonstrados por Green em [10].

Ainda aqui, $\mathcal{K}$ será um corpo e $\Lambda$ uma $\mathcal{K}$-álgebra com uma base multiplicativa $\mathcal{B}$ e uma ordem admissível $>$ em $\mathcal{B}$.

Seja $I$ um ideal de $\Lambda$ gerado por elementos da forma $b, b_{1}-b_{2}$, onde $b, b_{1}, b_{2} \in \mathcal{B}$, tal ideal é denominado um ideal 2 -nomial. 
Para relembrar, seja $X$ um conjunto. Se uma relação $\sim$ em $X$ satisfaz:

(1) $x \sim x$, para todo $x \in X$.

(2) Se $x \sim y$, então $y \sim x$, para todo $x, y \in X$.

(3) Se $x \sim y$ e $y \sim z$, então $x \sim z$, para todo $x, y, z \in X$.

dizemos que $\sim$ é uma relação de equivalência.

Proposiçāo 3.1 Existe uma correspondência biunívoca entre o conjunto de relações de equivalência em $\mathcal{B} \cup\{0\}$ e ideais 2-nomiais.

Prova. Seja $\sim$ uma relação de equivalência em $\mathcal{B} \cup\{0\}$. Definimos $I_{\sim}$ o ideal gerado por $b_{1}-b_{2}$ se $b_{1} \sim b_{2}$ e $b$ se $b \sim 0$.

Por outro lado se $I$ é um ideal 2 -nomial, seja $\sim_{I}$ a relação de equivalência dada por

(i) $b_{1} \sim_{I} b_{2}$ se $b_{1}-b_{2} \in I$

(ii) $b \sim_{I} 0$ se $b \in I$

Chamamos a relação de equivalência $\sim$ em $\mathcal{B} \cup\{0\}$ correspondente a. $I$ por relação associada (a I).

Lema 3.2 Seja I um ideal 2-nomial em $\Lambda$ com relação associada $\sim_{I}$. Então $\sum_{i=1}^{r} \alpha_{i} b_{i} \in I$, com $\alpha_{i} \in \mathcal{K}$ e $b_{i} \in \mathcal{B}$, se, e somente se, para cada classe [b] de $\sim_{I}$ temos $\sum_{b_{i} \in[b]} \alpha_{i} b_{i} \in I$.

Prova. Considere $\sum_{i=1}^{r} \alpha_{i} b_{i} \in \Lambda$. Se para cada classe de equivalência [b] a soma $\sum_{b_{i} \in[b]} \alpha_{i} b_{i} \in I$ é claro que $\sum_{i=1}^{r} \alpha_{i} b_{i} \in I$. 
Suponha que $x=\sum_{i=1}^{r} \alpha_{i} b_{i} \in I$. Então $x=\sum_{j=1}^{s} \beta_{j}\left(b_{j}-b_{j}^{\prime}\right)+\sum_{l=1}^{t} \gamma_{l} b_{l}$ com $b_{j}-b_{j}^{\prime}, b_{l} \in I$. Podemos reescrever $x$ como sendo:

$$
\begin{aligned}
x & =\sum_{i=1}^{r} \alpha_{i} b_{i} \\
& =\sum_{j=1}^{s} \beta_{j} b_{j}+\sum_{j=1}^{s}-\beta_{j} b_{j}^{\prime}+\sum_{l=1}^{t} \gamma_{l} b_{l}
\end{aligned}
$$

Fixamos então uma classe $[b]$ e vemos que

$$
\sum_{b_{i} \in[b]} \alpha_{i} b_{i}=\sum_{b_{j}, b_{j}^{\prime} \in[b]} \beta_{j}\left(b_{j}-b_{j}^{\prime}\right)+\sum_{b_{l} \in[b]} \gamma_{l} b_{l} .
$$

O resultado segue a partir dessa observação.

Teorema 3.3 Suponha que $S$ é uma $\mathcal{K}$-álgebra com base multiplicativa $\mathcal{C}$. Seja I ideal em $S$ e $\pi: S \rightarrow S / I$ a projeção canônica.

Seja $\mathcal{B}$ o conjunto dos elementos não nulos de $\pi(\mathcal{C})$. B é uma base multiplicativa de S/I se e somente se I é um ideal 2-nomial.

Prova. Primeiramente vamos mostar que se $I$ é um ideal 2-nomial, então $\mathcal{B}$ é uma base multiplicativa.

Sejam $\pi\left(c_{1}\right), \pi\left(c_{2}\right) \in \pi(\mathcal{C})$. Então $\pi\left(c_{1}\right) \pi\left(c_{2}\right)=\pi\left(c_{1} c_{2}\right) \in \pi(\mathcal{C})$ e $\pi(\mathcal{C})=$ $\mathcal{B} \cup\{0\}$.

Agora precisamos mostrar que $\mathcal{B}$ é uma $\mathcal{K}$-base de $S / I$, para isso é suficiente mostrar que os elementos de $\mathcal{B}$ são linearmente independentes.

Seja $\sum_{i=1}^{n} \alpha_{i} \pi\left(c_{i}\right)=0 \operatorname{com} \pi\left(c_{i}\right) \neq 0$ e distintos. Daí, $\sum_{i=1}^{n} \alpha_{i} c_{i} \in I$. Seja $\sim$ a relação em $\mathcal{C} \cup\{0\}$ associada a. $I$.

Pelo lema 3.2, para cada classe de equivalência [c], temos $\sum_{c_{i} \in[c]} \alpha_{i} c_{i} \in I$.

Se $c_{i} \in[c]$, então $\pi\left(c_{i}\right)=\pi(c)$. Logo os $c_{i}^{\prime} s$ estão em diferentes classes de equivalência, pois $\pi\left(c_{i}\right)$ são distintos. Assim $\alpha_{i} c_{i} \in I$ e concluimos que ou 
$c_{i} \in I$, o que não pode acontecer, pois estamos supondo $\pi\left(c_{i}\right) \neq 0$, ou $c_{i} \notin I$, o que implica em $\alpha_{i}=0$.

Portanto $\mathcal{B}$ é uma base multiplicativa de $S / I$.

Agora vamos mostrar que se $\mathcal{B}$ é uma base multiplicativa de $S / I$, então $I$ é um ideal 2-nomial.

Definimos a relação $\sim \operatorname{em} \mathcal{C} \cup\{0\}$ por $c \sim c^{\prime}$ se $\pi(c)=\pi\left(c^{\prime}\right)$. É fácil ver que o ideal 2-nomial correspondente a $\sim$ (pela proposição 3.1) é I. E isto completa a prova.

A partir desse ponto assumiremos que $\Lambda$ é uma $\mathcal{K}$-álgebra com unidade, mas não que $1 \in \mathcal{B}$, a menos que este seja o único idempotente. Assim, $1=\sum_{i=1}^{n} \alpha_{i} b_{i}$, com $\alpha_{i} \in \mathcal{K} \backslash\{0\}$ e $b_{i}$ distintos em $\mathcal{B}$.

Lema $3.4 O$ conjunto $\left\{b_{1}, \cdots, b_{n}\right\}$ é um conjunto de idempotentes ortogonais e cada $\alpha=1$.

Prova. Suponha que $b_{i} b_{j} \neq 0$ com $i \neq j$. Então $b_{i} b_{j}=b \in \mathcal{B}$. Assuma que $b \neq b_{i}$ (o caso em que $b \neq b_{j}$ é análogo). Então

$$
b_{i}=b_{i} \cdot 1=\sum_{j=1}^{n} \alpha_{j} b_{i} b_{j} .
$$

Assim, existe algum $l \neq j$ tal que $b_{i} b_{l}=b$, pois $b=b_{i} b_{j}$ deve ser cancelado por algum $b_{i} b_{l}$. Agora, ou $b_{l}>b_{j}$ ou $b_{j}>b_{l}$, mas em nenhum caso podemos ter $b_{i} b_{l}=b_{i} b_{j}=b$, pela ordem assumida. Portanto se $i \neq j$ tem-se $b_{i} b_{j}=0$.

$\operatorname{Logo} b_{i}=\alpha b_{i} b_{i}=\alpha b_{i}$ e portanto $\alpha=1$.

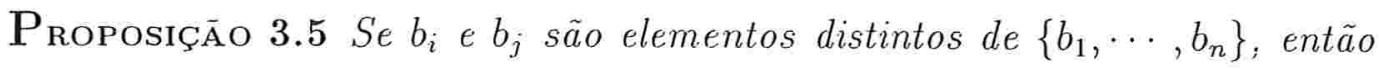
para todo $b \in \mathcal{B}, b_{i} b=b_{j} b$ implica $b_{i} b=b_{j} b=0$ e $b b_{i}=b b_{j}$ implica $b b_{i}=$ $b b_{j}=0$. 


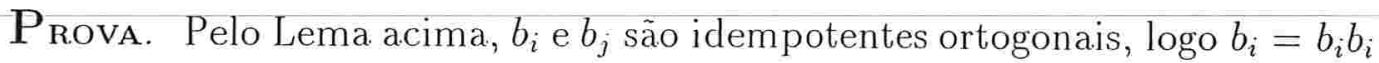
e $b_{i} b_{j}=b_{j} b_{i}=0$. Assim,

$$
b_{i} b=b_{j} b \Longrightarrow b_{i} b=b_{i} b_{i} b=b_{i} b_{j} b=0
$$

e

$$
b b_{i}=b b_{j} \Longrightarrow b b_{i}=b b_{i} b_{i}=b b_{j} b_{i}=0
$$

Como podemos observar, o conjunto de idempotentes $\left\{b_{1}, \cdots, b_{n}\right\}$ tais que $1=\sum_{i=1}^{n} b_{i}$ são elementos muito especiais de $\mathcal{B}$. Para distinguí-los, escreveremos $1=\sum_{i=1}^{n} v_{i} \operatorname{com} b_{i}$ substituidos pelos $v_{i}$.

Proposiçāo 3.6 Se $b \in \mathcal{B}$ então existem únicos $i, j$ tais que $v_{i} b=b$ e $b v_{j}=b$. Se $k \neq i$, então $v_{k} b=0$. Se $k \neq j$, então $b v_{k}=0$.

Prova. Como $b=b \cdot 1=\sum_{i=1}^{n} b v_{i}$, concluimos que $b v_{j}=b$ para algum $j$. Mas se $k \neq j$, como $v_{j} v_{k}=0, b v_{k}=0$. De forma análoga se mostra a segunda parte do resultado.

Se $b \in \mathcal{B}$, definimos $v_{i}$ como sendo a origem de $b, o(b)=v_{i}$, se $v_{i} b=b$. Da mesma forma, definimos $v_{j}$ por término de $b, t(b)=v_{j}$, se $b v_{j}=b$.

$\mathrm{O}$ próximo resultado mostra que os $v_{i}^{\prime} s$ tem a propriedade de minimalidade com respeito à. ordem $>$.

Lema 3.7 Se $b \in \mathcal{B}$ tal que o $(b)=v$ ou $t(b)=v$, então $b \geq v$.

Prova. Suponha que $o(b)=v$, assim $v b=b$, como $v$ é idempotente, temos $v v b=b$, no entanto a ordem que estamos assumindo é admissível, segue que $b \geq v$. 
Corolário 3.8 Se $b \in \mathcal{B} \backslash\left\{v_{1}, \cdots, v_{n}\right\}$, então $b^{2} \neq b$.

Prova. Se $b^{2}=0$ nada temos a fazer. Suponha que $b^{2} \neq 0$, então $o(b)=$ $t(b)=v$, pelo lema acima temos que $v<b$, utilizando a propriedade da ordem ser a.dmissível, temos $b=b v<b b$.

Lema $3.9 O$ conjunto $\mathcal{Q}_{0}=\left\{v_{1}, \cdots, v_{n}\right\}$ é um conjunto completo de idempotentes primitivos ortogonais para $\Lambda$.

Prova. Já temos visto que $\mathcal{Q}_{0}$ é um conjunto completo de idempotentes ortogonais, falta apenas mostar que ele é um conjunto primitivo.

Suponha, por absurdo, que $\mathcal{Q}_{0}$ não é um conjunto primitivo.

Seja $v_{i}=x+y$ para algum $i \in\{1, \cdots, n\}$, onde $x$ e $y$ são idempotentes ortogonais não nulos.

Agora, $x=\sum \alpha_{j} v_{j}+\sum \beta_{l} b_{l}$, com $\alpha, \beta \in \mathcal{K}$ e $b_{l} \in \mathcal{B}$.

Como $v_{i}=v_{i} x v_{i}+v_{i} y v_{i}$, vemos que $\alpha_{j}=0$ se $i \neq j$ e que se $\beta_{l} \neq 0$, então $o\left(b_{l}\right)=v_{i}=t\left(b_{l}\right)$. Logo,

$$
x=\alpha_{i} v_{i}+\sum \beta_{l} b_{l}
$$

e

$$
y=\left(1-\alpha_{i}\right) v_{i}-\sum \beta_{l} b_{l}
$$

Como $x y=0$ e $b_{l} b_{j} \neq v_{i}$, concluimos que $\alpha_{i}\left(1-\alpha_{i}\right)=0$.

Daí, podemos assumir que $\alpha_{i}=1$. Seja $b$ o menor $b_{l}$ ocorrendo em $\sum \beta_{l} b_{l}$.

Novamente usando que $x y=0, v_{i} b=b$ e que $b$ é menor que todos os produtos $b_{l} b_{k}$ não nulos, vemos que em $x y$ não se pode cancelar $v_{i} b$. Logo, todos os $\beta_{l}=0$. E isto completa a prova.

Definiçāo. 3.10 Seja $\mathcal{Q}_{1}=\left\{b \in\left(\mathcal{B} \backslash \mathcal{Q}_{0}\right): b\right.$ não pode ser escrito como produto $b_{1} b_{2}$ com $\left.b_{1}, b_{2} \in \mathcal{B} \backslash \mathcal{Q}_{0}\right\}$, ou seja, $\mathcal{Q}_{1}$ é o conjunto dos elementos indecomponiveis de $\mathcal{B}$. 
Proposiçāo 3.11 Seja $\Lambda$ uma $\mathcal{K}$-álgebra com base multiplicativa $\mathcal{B}$ e uma ordem admissivel $>$ em $\mathcal{B}$. Se $\mathcal{Q}_{0}$ e $\mathcal{Q}_{1}$ são os únicos subconjuntos de $\mathcal{B}$ definidos por $1=\sum_{v \in \mathcal{Q}_{0}} v$ e $\mathcal{Q}_{1}$ são os elementos indecomponíveis de $\mathcal{B} \backslash \mathcal{Q}_{0}$, então todo $b \in \mathcal{B} \backslash \mathcal{Q}_{0}$ é produto $b_{1} \ldots b_{r}$ com $b_{i} \in \mathcal{Q}_{1}$. Em particular; $\mathcal{Q}_{0} \cup \mathcal{Q}_{1}$ gera a base multiplicativa $\mathcal{B}$, como semigrupo.

Prova. Seja $b \in \mathcal{B} \backslash \mathcal{Q}_{0}$, se $b \in \mathcal{Q}_{1}$ nada temos que fazer. Suponha que $b \notin \mathcal{Q}_{0} \cup \mathcal{Q}_{1}$, então $b=b_{1} b_{2}$ para algum $b_{1}, b_{2} \in \mathcal{B} \backslash \mathcal{Q}_{0}$. Como $b=o\left(b_{1}\right) b_{1} b_{2}$ e $b=b_{1} b_{2} t\left(b_{2}\right)$, vemos que $b>b_{1}$ e $b>b_{2}$, pela ordem assumida.

Se $b_{1}, b_{2} \in \mathcal{Q}_{1}$ terminamos aqui a demonstração. Se não, continuamos o processo obtendo $b_{i}=b_{i_{1}} \ldots b_{i_{n}}, \operatorname{com} b_{i_{j}} \in \mathcal{B} \backslash \mathcal{Q}_{0}$.

Como a ordem $>$ é total, temos uma cadeia descendente $b>b_{i_{s_{1}}}>\cdots$. Mas > é uma boa ordem, logo esse processo estabiliza (teorema 1.12), isto é, cada $b_{i_{j}}$ é um produto finito de elementos de $\mathcal{Q}_{0} \cup \mathcal{Q}_{1}$, terminando assim a. prova.

Agora, seja $\mathcal{Q}$ o quiver com conjunto de vértices $\mathcal{Q}_{0}$ e conjunto de flechas $\mathcal{Q}_{1}$, isto é, se $b \in \mathcal{Q}_{1}$, o vemos como sendo uma flecha de origem $o(b)$ e término $t(b)$. Chamamos $\mathcal{Q}$ o quiver associado a $\mathcal{B}$.

Teorema 3.12 Seja $\Lambda$ uma $\mathcal{K}$-álgebra com uma base multiplicativa $\mathcal{B}$ e uma ordem admissível $>$ em $\mathcal{B}$. Seja $\mathcal{Q}$ o quiver associado a $\mathcal{B}$. Então existe um epimorfismo de $\mathcal{K}$-álgebras

$$
\varphi: \mathcal{K Q} \rightarrow \Lambda
$$

tal que:

(i) S'e $p$ é um caminho em $\mathcal{Q}$, então $\varphi(p) \in \mathcal{B} \cup\{0\}$.

(ii) Se $b \in \mathcal{B}$, então existe um caminho $p \in \mathcal{Q}$ tal que $\varphi(p)=b$.

(iii) O núcleo de $\varphi$ é um ideal 2-nomial. 
Prova. Seja $\Lambda$ uma $\mathcal{K}$-álgebra com uma base multiplicativa $\mathcal{B}$ e ordem admissível > nessa base. Temos visto que existem únicos subconjuntos $\mathcal{Q}_{0}$ e $\mathcal{Q}_{1}$ de $\mathcal{B}$. Além disso, pelo lema $3.9, \mathcal{Q}_{0}$ é um conjunto completo de idempotentes primitivos ortogonais. Seja $\mathcal{Q}$ o quiver associado a $\Lambda$.

Definimos o homomorfismo de álgebras $\varphi: \mathcal{K Q} \rightarrow \Lambda$, enviando os vértices $v$ de $\mathcal{Q}$ ao seu correspondente em $\mathcal{Q}_{0} \subset \mathcal{B}$ e as flechas de $\mathcal{Q}$ no elemento correspondente em $\mathcal{Q}_{1} \subset \mathcal{B}$. Por construção, caminhos de $\mathcal{Q}$ são enviados a elementos de $\mathcal{B} \cup\{0\}$ e pela proposição 3.11 , temos que $\varphi$ é sobrejetiva.

Note que a imagem da base multiplicativa dos caminhos de $\mathcal{Q}$ pela $\varphi$ é $\mathcal{B} \cup\{0\}$. Assim, pelo teorema 3.3, concluimos que o núcleo de $\varphi$ é um ideal 2 -nomial de $\mathcal{Q}$, completando assim a prova.

Sabemos que dada uma $\mathcal{K}$-álgebra $\Lambda$ com uma base $\mathcal{B}$ multiplicativa e uma ordem admissível $>$ em $\mathcal{B}$, existe um quiver $\mathcal{Q}$ tal que $\Lambda=\mathcal{K} \mathcal{Q} / I$, onde $I$ é um ideal 2-nomial. Surge então a seguinte pergunta: quais as condições necessárias e suficientes sobre o ideal 2-nomial I, para o quociente de álgebra de caminhos $\mathcal{K} \mathcal{Q} / I$ tenha uma base $\mathcal{B}$ multiplicativa com uma ordem admissivel $>$ ?

O que se sabe até agora é que a afirmativa não vale em geral para todos os ideais 2-nomiais, como podemos ver no exemplo abaixo:

Exemplo. Seja $\mathcal{Q}$ o seguinte quiver:

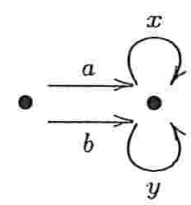

Seja o ideal 2-nomial $I=\left\langle a x^{n}-b, a-b y^{m}: m, n \in \mathbb{N}^{*}>\right.$. Considere em $\Lambda=\mathcal{K} \mathcal{Q}$ a $\mathcal{K}$-base formada por todos os caminhos de $\mathcal{Q}$. Para $\Lambda / I$ definimos a. seguinte $\mathcal{K}$-base $\overline{\mathcal{B}}=\mathcal{B} \backslash\left\{b x^{n}, a y^{m}, a x^{n}, b y^{m}, a x^{n} y^{m}, b y^{m} x^{n}: m, n \in \mathbb{N}^{*}\right\}$.

Suponha que $\overline{\mathcal{B}}$ tenha uma ordem admissível e que $a>b$. Como $x<x y$, 
multiplicamos pela esquerda por $a$, e obtemos a seguinte relação:

$$
\begin{aligned}
a(x) & <a(x y) \\
b & <(a x) y \\
b & <b y \\
b & <a
\end{aligned}
$$

O que não poderia acontecer em tal ordem.

A opção que nos resta é $a<b$, no entanto se tomarmos a relação $y<x y$ e multiplicarmos pela esquerda por $a$ chegaremos ao absurdo de que $b<a$. Portanto, para este quociente é impossível se definir uma ordem admissível.

\subsection{Homogeneização}

Em [3] é apresentado o processo de homogeneização para o caso comutativo, no anel de polinômios com $n$ variáveis.

Sabendo que o anel de polinômios em $n$ variáveis comutativas é um caso particular das álgebras de caminhos e que toda álgebra com unidade com a teoria de bases de Gröbner é isomorfa ao quociente de uma álgebra de caminhos, teorema 3.12 , veio-nos a pergunta se o mesmo processo, e também quais os resultados, podia-se aplicar às álgebras de caminhos. Nesta seção procuramos responder a esta pergunta com a versão, do caso não comutativo, para as álgebras de caminhos $\mathcal{K} \mathcal{Q} / I$, onde $I$ é um ideal bilateral de $\mathcal{K} \mathcal{Q}$.

Sejam $\mathcal{K}$ um corpo e $Q$ um quiver finito. Seja $\Lambda=\mathcal{K} Q / I$ a álgebra de caminhos associada a $Q$ e $I$ um ideal bilateral de $\mathcal{K} Q$. Considere em $\Lambda$ uma base multiplicativa $\mathcal{B}$ e $>$ uma ordem admissível em $\mathcal{B}$.

Definimos então $\tilde{Q}$ acrescentando a $Q$ um laço em cada vértice e a partir daí a $\mathcal{K}$-álgebra $\Lambda^{\prime}=\mathcal{K} \tilde{Q} / \tilde{I}$, onde $\tilde{I}=\langle I, \alpha Z-Z \alpha\rangle$ como ideal bilateral de $\mathcal{K} \tilde{Q}, \alpha \in \mathcal{Q}_{1}$ e $Z=\sum_{i \in\left|Q_{0}\right|} l_{i}$, aqui $l_{i}$ representa cada um dos novos laços acrescidos a $Q$. Chamaremos $\Lambda^{\prime}$ a álgebra extendida por laços de $\Lambda$. 
Tomamos para $\Lambda^{\prime}$ a seguinte base $\tilde{\mathcal{B}}=\left\{Z^{n} b: b \in \mathcal{B}\right.$ e $\left.n \geq 0\right\}$.

Tanto $\Lambda$ quanto $\Lambda^{\prime}$ são finitamente geradas como $\mathcal{K}$-álgebras, logo $\mathcal{B}$ e $\tilde{\mathcal{B}}$ são finitamente gerados como semigrupos.

Assim, fixemos para $\mathcal{B}$ um conjunto gerador minimal $U$, como semigrupo. Para $\tilde{\mathcal{B}}$, tomemos $\tilde{U}=U \cup\left\{l_{i}: i \in\left|\mathcal{Q}_{0}\right|\right\}$.

Definimos por $\ell(b)$ o comprimento de $b \in \mathcal{B}$ (respectivamente $\tilde{\mathcal{B}}$ ) o menor $n \in \mathbb{N}$ tal que $b=b_{1} b_{2} \cdots b_{n}$ com $b_{i} \in U$ (respectivamente $\left.\tilde{U}\right)$. Em $f \in \Lambda$ $\left(\Lambda^{\prime}\right)$, definimos o comprimento de $f$ por $\ell(f)=\max \{\ell(b): b \in \mathcal{B}(\tilde{\mathcal{B}})$ ocorra em. $f\}$. Diremos que um elemento $f=\sum_{i=1}^{n} \lambda_{i} b_{i}$, com $\lambda_{i} \in \mathcal{K}$ e $b_{i} \in \mathcal{B}(\tilde{\mathcal{B}})$, é homogêneo se $\ell(f)=\ell\left(b_{i}\right)$ para todo $1 \leq i \leq n$.

Um ideal $I$ é homogêneo se pode ser gerado por elementos homogêneos.

Definimos em $\tilde{\mathcal{B}}$ a ordem

$$
\begin{gathered}
e_{i} \prec l_{j} \prec b, \text { para todo } i, j \in\left|\mathcal{Q}_{0}\right| \text { e } b \in \mathcal{B} \text { e } \\
Z^{n} b_{1} \prec Z^{m} b_{2} \text { se }:\left\{\begin{array}{l}
\text { se } b_{1}<b_{2} \text { em } \mathcal{B} \text { ou } \\
\text { se } b_{1}=b_{2} \text { em } \mathcal{B} \text { e } n<m
\end{array}\right.
\end{gathered}
$$

Vamos mostrar que a ordem acima é de fato uma ordem admissível. Sejam $Z^{n} b_{1}, Z^{m} b_{2}, Z^{r} b_{3}, Z^{s} b_{4} \in \tilde{\mathcal{B}}$, então:

(1) se $Z^{n} b_{1} \prec Z^{m} b_{2}$ e $Z^{n} b_{1} Z^{r} b_{3}$ e $Z^{m} b_{2} Z^{r} b_{3}$ são não nulos, temos que, se $b_{1}<b_{2}$ então $b_{1} b_{3}<b_{2} b_{3}$. Agora, se $b_{1}=b_{2}, n<m$ e portanto $b_{1} b_{3}=b_{2} b_{3}$ e $n+r<m+r$. Assim, $Z^{n} b_{1} Z^{r} b_{3} \prec Z^{m} b_{2} Z^{r} b_{3}$.

(2) da mesma forma, se $Z^{n} b_{1} \prec Z^{m} b_{2}$, então $Z^{r} b_{3} Z^{n} b_{1} \prec Z^{r} b_{3} Z^{m} b_{2}$, se os produtos são não nulos.

(3) se $Z^{n} b_{1}=Z^{m} b_{2} Z^{r} b_{3} Z^{s} b_{4}=Z^{m+r+s} b_{2} b_{3} b_{4}$, temos que $r \leq n$ e $b_{3} \leq b_{1}$ $\operatorname{logo} Z_{r} b_{3} \preceq Z^{n} b_{1}$.

Portanto a ordem $\prec$ definida acima é uma ordem admissível. 
Definiçāo. 3.13 Para $f=\sum_{i=1}^{m} \lambda_{i} b_{i} \in \Lambda$, definimos o homogeneizado de $f$ em $\Lambda^{\prime}$ por $f^{*}=\sum_{i=1}^{m} \lambda_{i} Z^{\ell(f)-\ell\left(b_{i}\right)} b_{i}$.

Note que, para todo $f \in \Lambda$, o homogeneizado de $f$ é homogêneo.

Lema 3.14 Para todo $f, g \in \Lambda$, temos $Z^{k}(f g)^{*}=f^{*} g^{*}$, onde $k=\ell(f)+$ $\ell(g)-\ell(f g)$.

Prova. Sejam $f=\sum_{i=1}^{n} \lambda_{i} b_{i}$ e $g=\sum_{j=1}^{m} \beta_{j} b_{j}, \operatorname{com} \lambda_{i}, \beta_{j} \in \Lambda$ e $b_{i}, b_{j} \in B$. Como $\ell(f)+\ell(g) \geq \ell(f g)$, tomamos $k=\ell(f)+\ell(g)-\ell(f g)$. Daí,

$$
\begin{aligned}
f^{*} g^{*} & =\left(\sum_{i=1}^{n} \lambda_{i} b_{i}\right)^{*}\left(\sum_{j=1}^{m} \beta_{j} b_{j}\right)^{*} \\
& =\left(\sum_{i=1}^{n} \lambda_{i} Z^{\ell(f)-\ell\left(b_{i}\right)} b_{i}\right)\left(\sum_{j=1}^{m} \beta_{j} Z^{\ell \ell(g)-\ell\left(b_{j}\right)} b_{j}\right) \\
& =\sum_{i, j} \lambda_{i} \beta_{j} Z^{(\ell(f)+\ell(g))-\left(\ell\left(b_{i}\right)+\ell\left(b_{j}\right)\right)} b_{i} b_{j} \\
& =\sum_{i, j} \lambda_{i} \beta_{j} Z^{\ell(f g)-\ell\left(b_{i} b_{j}\right)} Z^{k} b_{i} b_{j} \\
& =Z^{k}\left(\sum_{i, j} \lambda_{i} \beta_{j} b_{i} b_{j}\right)^{*} \\
& =Z^{k}\left(\left(\sum_{i=1}^{n} \lambda_{i} b_{i}\right)\left(\sum_{j=1}^{m} \beta_{j} b_{j}\right)\right)^{*} \\
& =Z^{k}(f g)^{*}
\end{aligned}
$$


Definimos agora a seguinte aplicação entre as álgebras $\Lambda^{\prime}$ e $\Lambda$ :

$$
\varphi: \Lambda^{\prime} \rightarrow \Lambda
$$

que a cada elemento $Z^{n} b \in \tilde{\mathcal{B}}$ associa o elemento $b \in \mathcal{B}$, para todo $n \in \mathbb{N}$. Note que, para cada $b \in \mathcal{B}$, existe $Z b \in \tilde{\mathcal{B}}$ tal que $\varphi(Z b)=b$. Dessa forma, temos que $\varphi$ estendida por linearidade a toda $\Lambda^{\prime}$ é de fato um epimorfismo de álgebras. Note que $\varphi\left(\sum_{i=1}^{n} Z^{k_{i}} e_{i}\right)=1$, para todo $k_{i} \in \mathbb{N}$ e que $\operatorname{ker}(\varphi)=$ $\langle Z-1\rangle$.

Para simplificar a notação, usaremos $g_{*}=\varphi(g)$ para todo $g \in \Lambda^{\prime}$.

Lema 3.15 Para todo $f \in \Lambda$ temos $\left(f^{*}\right)_{*}=f$.

Prova. Seja $f=\sum_{i=1}^{n} \lambda_{i} b_{i}$, com $\lambda_{i} \in \Lambda$ e $b_{i} \in \mathcal{B}$. Note que

$$
\begin{aligned}
\left(f^{*}\right)_{*} & =\left(\sum_{i=1}^{n} \lambda_{i} Z^{\ell(f)-\ell\left(b_{i}\right)} b_{i}\right)_{*} \\
& =\sum_{i=1}^{n}\left(\lambda_{i} Z^{\ell(f)-\ell\left(b_{i}\right)} b_{i}\right)_{*} \\
& \left.=\sum_{i=1}^{n} \lambda_{i}\left(Z^{\ell(f)-\ell\left(b_{i}\right)}\right)\right)_{*}\left(b_{i}\right)_{*} \\
& =\sum_{i=1}^{n} \lambda_{i} b_{i} \\
& =f
\end{aligned}
$$

Lema 3.16 Seja $g \in \Lambda^{\prime}$ homogêneo de comprimento d e seja $d^{\prime}=\ell\left(g_{*}\right)$. Então $d^{\prime} \leq d$ e $g=Z^{d-d^{\prime}}\left(g_{*}\right)^{*}$.

Prova. A desigualdade $d^{\prime} \leq d$ é imediata da definição de $g_{*}$. Seja $m \in$ $\operatorname{supp}_{\tilde{\mathcal{B}}}(g), m=t Z^{i}$, com $t \in \mathcal{B}$. Assim o monômio $m_{*} \in \operatorname{supp}_{\mathcal{B}}\left(g_{*}\right)$ correspon- 
dente a $m$ é $t$. Como $\ell(t)=d-i$ o monômio em $\operatorname{supp}\left(\left(g_{*}\right)^{*}\right)$ correspondente a. $m_{*}$ é $t Z^{d^{\prime}-(d-i)}$. Logo $Z^{d-d^{\prime}}\left(g_{*}\right)^{*}=g$.

Definiçāo. 3.17 Sejam. $F \subset \Lambda$ G $\mathcal{G} \subset \Lambda^{\prime}$, definimos por

$$
\begin{aligned}
& F^{*}=\left\{f^{*}: f \in F\right\} \\
& \mathcal{G}_{*}=\left\{g_{*}: g \in \mathcal{G}\right\}
\end{aligned}
$$

Lema 3.18 Seja $f \in \Lambda$. Então $\ell(f)=\ell\left(f^{*}\right)$.

Prova. Considere $f=\sum_{i=1}^{m} \lambda_{i} b_{i} \operatorname{com} \lambda_{i} \in \mathcal{K}$ e $b_{i} \in \mathcal{B}$, base de $\Lambda, 1 \leq i \leq$ $m$.

Por definição temos que $f^{*}=\sum_{i=1}^{m} \lambda_{i} Z^{l(f)-l\left(b_{i}\right)} b_{i}$. Para todo somando de $f^{*}$ temos:

$$
\ell\left(Z^{\ell(f)-\ell\left(b_{i}\right)} b_{i}\right)=\ell\left(Z^{\ell(f)-\ell\left(b_{i}\right)}\right)+\ell\left(b_{i}\right)=\left(\ell(f)-\ell\left(b_{i}\right)\right)+\ell\left(b_{i}\right)=\ell(f)
$$

Logo, $\ell\left(f^{*}\right)=\max \left\{\ell\left(Z^{\ell(f)-\ell\left(b_{i}\right)} b_{i}\right): 1 \leq i \leq m\right\}=\ell(f)$

Lema 3.19 Seja $F=\left\{f_{i}\right\}_{i \in \mathcal{I}}$ um subconjunto de $\Lambda$, não necessariamente finito, $e f=\sum_{i=1}^{m} r_{i} f_{i} s_{i} \in\langle F\rangle$. Se $d=\max \left\{\ell\left(r_{i} f_{i} s_{i}\right): 1 \leq i \leq m\right\} e$ $d^{\prime}=\ell(f)$. Então $Z^{d-d^{\prime}} f^{*} \in\left\langle F^{*}\right\rangle$.

Prova. Pelo Lema 3.18, temos que $d=\max \left\{\ell\left(\left(r_{i} f_{i} s_{i}\right)^{*}\right): 1 \leq i \leq m\right\}$. Tome $k_{i}=\ell\left(r_{i}\right)+\ell\left(f_{i}\right)+\ell\left(s_{i}\right)-\ell\left(r_{i} f_{i} s_{i}\right), 1 \leq i \leq m$.

Seja $\bar{f}=\sum_{i=1}^{m} Z^{d-\ell\left(r_{i} f_{i} s_{i}\right)} Z^{k_{i}}\left(r_{i} f_{i} s_{i}\right)^{*}=\sum_{i=1}^{m}\left(Z^{d-\ell\left(r_{i} f_{i} s_{i}\right)} r_{i}\right)^{*} f_{i}^{*} s_{i}^{*}$, pelo lema 3.14. Logo, $\bar{f} \in\left\langle F^{*}\right\rangle$ e é homogêneo (por construção) com $d^{\prime \prime}=\ell(\bar{f}) \leq d$. 
Além disso, usando o lema 3.14 e o lema 3.15 , temos

$$
\begin{aligned}
\bar{f}_{*} & =\sum_{i=1}^{m}\left(Z^{d-\ell\left(r_{i} f_{i} s-i\right)} Z^{k_{i}}\left(r_{i} f_{i} s_{i}\right)^{*}\right)_{*} \\
& =\sum_{i=1}^{m}\left(Z^{d-\ell\left(r_{i} f_{i} s_{i}\right)}\right)_{*}\left(r_{i}^{*}\right)_{*}\left(f_{i}^{*}\right)_{*}\left(s_{i}^{*}\right)_{*} \\
& =\sum_{i=1}^{m} r_{i} f_{i} s_{i}=f
\end{aligned}
$$

Usando o Lema 3.16, podemos concluir que

$$
\bar{f}=Z^{d^{\prime \prime}-d^{\prime}}\left(\bar{f}_{*}\right)^{*}=Z^{d^{\prime \prime}-d^{\prime}} f^{*}
$$

Como $d^{\prime \prime} \leq d$, finalmente temos:

$$
Z^{d-d^{\prime}} f^{*}=Z^{d-d^{\prime \prime}} Z^{d^{\prime \prime}-d^{\prime}} f^{*}=Z^{d-d^{\prime \prime}} \bar{f} \in\left\langle F^{*}\right\rangle
$$

Lema 3.20 Seja $F$ um subconjunto de $\Lambda$. Então $\left(\left\langle F^{*}\right\rangle\right)_{*}=\langle F\rangle$.

Prova. Seja $f \in\langle F\rangle$. Pelo Lema $3.19, Z^{k} f^{*} \in\left\langle F^{*}\right\rangle$, para algum $k \in \mathbb{N}$, e assim

$$
f=\left(f^{*}\right)_{*}=\left(Z^{k} f^{*}\right)_{*} \in\left(\left\langle F^{*}\right\rangle\right)_{*}
$$

Por outro lado, se $g \in\left\langle F^{*}\right\rangle$, digamos $g=\sum_{i=1}^{m} r_{i}\left(f_{i}\right)^{*} s_{i} \operatorname{com} f_{i} \in F$ e $r_{i}, s_{i} \in \Lambda^{\prime}$ para $1 \leq i \leq m$, temos

$$
\begin{aligned}
g_{*} & =\left(\sum_{i=1}^{m} r_{i}\left(f_{i}\right)^{*} s_{i}\right)_{*} \\
& =\sum_{i=1}^{m}\left(r_{i}\right)_{*}\left[\left(f_{i}\right)^{*}\right]_{*}\left(s_{i}\right)_{*} \\
& =\sum_{i=1}^{m}\left(r_{i}\right)_{*} f_{i}\left(s_{i}\right)_{*}
\end{aligned}
$$

Logo, $g_{*} \in\langle F\rangle$. 
Teorema 3.21 Seja F um subconjunto de $\Lambda$ e seja $\mathcal{G} \subset \Lambda^{\prime}$ homogêneo. Se $\mathcal{G}$ é uma base de Gröbner para $\left\langle F^{*}\right\rangle$, então $\mathcal{G}_{*}$ é uma base de Gröbner de $\langle F\rangle$.

Prova. Suponha que $\mathcal{G}$ é uma base de Gröbner para $\left\langle F^{*}\right\rangle$. Vamos recorrer à definição de base de Gröbner.

Como $\mathcal{G}_{*} \subset\langle F\rangle$, então $\left\langle\operatorname{Tip}\left(\mathcal{G}_{*}\right)\right\rangle \subseteq\langle\operatorname{Tip}(\langle F\rangle)\rangle$, resta-nos verificar que $\langle\operatorname{Tip}(\langle F\rangle)\rangle \subseteq\left\langle\operatorname{Tip}\left(\mathcal{G}_{*}\right)\right\rangle$, ou seja, dado $f \in\langle F\rangle$ existe $g_{*} \in \mathcal{G}_{*}$ tal que Tip $\left(g_{*}\right)$ divide Tip $(f)$.

Seja $f \in\langle F\rangle$, podemos escrever $f=\sum_{i=1}^{m} \lambda_{i} b_{i}$, onde $\lambda_{i} \in \mathcal{K}$ e $b_{i} \in \mathcal{B}$ para $1 \leq i \leq m$.

Sem perda de generalidade, suponha que $\operatorname{Tip}(f)=b_{1}$. Note que $\ell\left(b_{1}\right)=$ $\ell(f)$, daí

$$
f^{*}=\lambda_{1} b_{1}+\sum_{i=2}^{m} \lambda_{i} Z^{\ell(f)-\ell\left(b_{i}\right)} b_{i}
$$

segue então pela ordem que $\operatorname{Tip}\left(f^{*}\right)=b_{1}=\operatorname{Tip}(f)$.

Pelo lema 3.19, existe $k \in \mathbb{N}$ tal que $h=Z^{k} f^{*} \in\left\langle F^{*}\right\rangle$. Pela observação acima, $\operatorname{Tip}(h)=Z^{k} \operatorname{Tip}(f)$.

Como $\mathcal{G}$ é uma base de Gröbner para $\left\langle F^{*}\right\rangle$, existe $g \in \mathcal{G}$ tal que $\operatorname{Tip}(h)=$ $Z^{k_{r}} r \operatorname{Tip}(g) Z^{k_{s}} s$, para algum $Z^{k_{r}} r, Z^{k_{s}} s \in \tilde{\mathcal{B}}$. Tip $(g) \in \tilde{\mathcal{B}}$, logo $\operatorname{Tip}(g)=Z^{k_{g}} b$ para algum $b \in \mathcal{B}$ e $k_{g} \in \mathbb{N}$.

Daí, Tip $(h)=Z^{k} b_{1}=Z^{k_{r}} r T i p(g) Z^{k_{s}} s=Z^{k_{r}} r Z^{k_{g}} b Z^{k_{s}} s=Z^{k_{r}+k_{g}+k_{s}} r b . s$.

Por hipótese $g$ é homogêneo, assim $\operatorname{Tip}(g)=Z^{k_{g}} b$ é tal que, para todo $Z^{t} b_{t} \neq$ Tip $(g)$ ocorrendo em $g$, temos que ou $k_{g}<t$ o que implica em $b>b_{t}$ e $\operatorname{assim} \operatorname{Tip}\left(g_{*}\right)=b$. Logo Tip $(f)=r \operatorname{Tip}\left(g_{*}\right) s$, e assim $\mathcal{G}_{*}$ é uma base de Gröbner para $\langle F\rangle$. 


\section{Referências Bibliográficas}

[1] Assem, I., Simsom, D., Skowronski, A., Representation Theory of Finite Dimensional Algebras, preprint.

[2] Auslander, M., Reiten, I., Smal $\phi$, S., Representation Theory of Artin Algebras, vol 36 of Cambridge Studies in Advanced Mathematics, Cambridge University Press.

[3] Becker, T., Weispfenning, V., Gröbner Bases, A Computational Approach to Commutative Algebra, Graduate Texts in Mathematics, SpringerVerlag. 1993.

[4] Benson, B. J., Representations and Cohomology, Cambridge University Press. 1991.

[5] Bergman, G. M., The Diamond Lemma for ring theory, Adv. in Math. $29,1978,178-218$.

[6] Buchberger, An Algorithm for Finding a Basis for the Residue Class Ring of a Zero-Dimensional Ideal, Ph.D. Thesis, University of Innsbruck, 1965.

[7] Farkas, D. R., Feustel, C. D., Green, E. L., Synergy in the Theories of Gröbner Bases and Path Algebras, Can. J. Math., 45, 1993, 727-739. 
[8] Feustel, C. D., Green, E. L., Kirkman, E., Kuzmanovich, J., Constructing Projective Resolutions, Comm. in Alg., 21, 1993, 1869-1887

[9] Green, E. L., Multiplicative Bases, Gröbner Bases, and Right Gröbner Bases, J. Symbolic Computation, 29, 2000, n.4-5, 601-623.

[10] Green, E. L., Noncommutative Gröbner Bases and Projectives Resolutions, In Michler and Schneider, eds, Proceedings of the Euroconference Computational Methods for Representations of Groups and Algebras, Essen, 1997, vol. 173 of Progress in Mathematics, 29-60. Basel, Bikhaüser Verlag. 